\title{
Internal Relationship and Impact Path Between Innovation and Entrepreneurship: Based on China's High-tech Industry
}

\author{
Kai Zhao", Lixiang Wang \\ School of statistics, Huaqiao University, China \\ Email:kzhao_kai@163.com
}

\begin{abstract}
Innovation is the source of entrepreneurship, entrepreneurship is the value embodiment of innovation, and the two are inseparable. At a time when dividends such as population, reform and opening up, and resources and environment are gradually disappearing, China urgently needs to accelerate scientific and technological innovation to support economic development, incubate scientific and technological enterprises, and ease labor market pressure with technological progress and efficiency improvement. This paper focuses on China's high-tech industry, which is dominated by scientific and technological innovation. Starting from the overall, local, and regional perspectives, it organically integrates the traditional DEA, similar SFA, Malmquist index decomposition, chain multiple intermediary effect, and other multilevel research through cross-level analysis. Based on the research foundation of innovation efficiency after eliminating environmental and random factors, it deeply discusses the action path and impact mechanism of "double innovation" and provides targeted policy recommendations for the government and relevant local departments. The research confirms that the total effect of innovation on entrepreneurship is always positive, i.e., promoting "people-to-people innovation" is conducive to promoting "mass entrepreneurship" whether it is analyzed from the whole or from the part.
\end{abstract}

Keywords: innovation, high-tech industry, DEA, chain multiple mediation effect analysis

\section{Introduction}

Innovation is the source of starting a business. In the process of starting a business, one needs to have a continuous and vigorous sense of innovation before one can produce creative ideas or schemes and explore new modes and outlets. Entrepreneurship is the embodiment of the value of innovation. The value of innovation mainly lies in transforming technology into productivity, and the fundamental way to realize this transformation is entrepreneurship. It can be seen that innovation and entrepreneurship are inseparable. Studying the internal relationship and mechanism between the two can not only provide an important theoretical basis and guidance for China to promote "double innovation" and prevent "deviation and deviation" in the middle of the process but also lead enterprises to carryout efficient innovation input and output, accelerate enterprises to adapt to the new normal, and provide power and guarantee for promoting innovationdriven strategy in depth.

With the accelerated process of economic globalization and the increasingly complicated and steep international market, China, as the largest developing country, urgently needs to play the role of a leader in scientific and technological innovation to support economic development, incubate scientific and technological enterprises, and relieve the pressure on the labor market at a time when its low-cost comparative advantages in production factors such as demographic dividend, reform and opening-up dividend, resources and environment dividend, and the resulting labor force no longer exist. Therefore, this paper focuses on China's high-tech industry, which is dominated by scientific and technological innovation. Starting from the overall, local, and even regional perspectives, it attempts to organically integrate the traditional DEA, similar SFA, Malmquist index decomposition, chain multiple intermediary effect, and other multilevel research. Based on the research on innovation efficiency after eliminating environmental and random factors, it comprehensively and deeply discusses the action path and impact mechanism of "double innovation"and provides targeted policy recommendations for the government and relevant local departments.

This article will summarize and review the relevant literature from three aspects: innovation, entrepreneurship and innovation and entrepreneurship. 
First, about innovation. Scholars at home and abroad have conducted in-depth research on the innovation efficiency of China's high-tech industry from different perspectives. From the viewpoint of spatial economics, nonparametric DEAMalmquist index method is used to explore the main factors affecting the innovation efficiency of China's high-tech industries ${ }^{[1]}$. Starting from the perspective of industry comparison, the panel stochastic frontier method (SFA) is used to evaluate the innovation efficiency of China's high-tech industry, and the influencing factors of innovation efficiency are analyzed ${ }^{[2]}$. The research proves that institutional innovation and the technicality of scientific researchers are the keys to improve the innovation efficiency of China's high-tech industry. From the perspective of value chain, Xiao et al. ${ }^{[3]}$ empirically tested various factors that can affect innovation efficiency by using two-stage chain-linked DEA and Tobit models. The research shows that government support, financial environment, and other factors have a significant impact on innovation efficiency. And Chiu et al. ${ }^{[4]}$, based on the value chain perspective, constructed a model combining research and development investment and operation process and measured the innovation efficiency of China's high-tech industry research and development value chain. In addition, there are many scholars from the SCP analysis perspective of industrial organization theory to study the impact of enterprise size, market power, technological opportunities, market process, and other factors on innovation efficiency ${ }^{[5,6]}$.

Second, about starting a business. Entrepreneurship is a social activity involving various phenomena, and scholars at home and abroad have conducted in-depth research on it from different angles such as economics, sociology, and management. Chen and Yang ${ }^{[7]}$ based on microeconomics theory and from the perspective of industry differences analyzed the differences in the impact of various types of entrepreneurship on enterprise R\&D incentives. Cao et al. ${ }^{[8]}$ based on the social learning theory put forward a model of imitating entrepreneurial decision-making mechanism of high-tech industrial clusters. The study found that entrepreneurs implement entrepreneurial decision-making behavior under the stimulus of entrepreneurial motivation through observation and learning. Wang and Xue ${ }^{[9]}$ set out from the theory of organizational learning to construct the framework of intellectual property entrepreneurship under the background of China's transformation Intellectual Property Rights - Patent and upgrading and take high-tech industry as an example to carry out empirical analysis to perfect the theory of intellectual property entrepreneurship. Franco and Filson ${ }^{[10]}$ based on the innovation theory, on the basis of the knowledge spillover effect mechanism of employee flow diffusion, allow employees of current companies to imitate the behavior of their former employers, theoretically analyze the equilibrium conditions for Pareto optimality, and find out through simulation that derivative enterprises established by R\&D personnel usually have higher R\&D willingness and efficiency than other types of entrepreneurs.

Third, about innovation and entrepreneurship. At present, there are few studies abroad that can consider innovation and entrepreneurship together, and there are two completely opposite views. One view is that entrepreneurship is the process of realizing innovation, and innovation is the essential core of entrepreneurship ${ }^{[1]}$. From this point of view, innovation and entrepreneurship have an internal connection mechanism. However, another view is that entrepreneurship may involve innovation process, but it may not include innovation behavior ${ }^{[12]}$, and there is no inevitable connection between innovation and entrepreneurship. Domestic scholars, mainly based on high-tech industries, empirically study the interaction between innovation and entrepreneurship. Based on the questionnaire survey data of China's high-tech industrial development zones, taking into account the dual effects of innovation activities and entrepreneurial activities within innovation clusters on the development of innovation clusters, this paper makes an empirical analysis on the development of entrepreneurship, innovation, and innovation clusters in China's high-tech industries ${ }^{[13]}$. Jin and Xie ${ }^{[14]}$, based on the actual development of China's high-tech industry, explored the role of government in the process of enterprise innovation and entrepreneurship.

Compared with previous literatures, the innovation of this paper mainly includes the following two aspects: from the perspective of research content, the relationship between innovation and entrepreneurship studied in this paper is a relatively blank research field, and the relevant research results at home and abroad are less, and the conclusions are quite different, while the research on the internal mechanism of innovation's impact on entrepreneurship combined with intermediary effect is even less, so this paper is novel in content. From the perspective of research methods, this paper firstly decomposes total factor productivity (TFP) based on DEA model excluding environmental and random factors and then uses chain multiple intermediary effect model to analyze the influencing process and mechanism among various variables and further analyzes the relationship between innovation and entrepreneurship in high-tech industries. Compared with the previous literature, this paper emphasizes the application of cross-level analysis in methodology; organically combines the traditional DEA, similar SFA, Malmquist index decomposition, chain multiple intermediary effect, and other multilevel related researches; and further discusses the action path and influencing mechanism of double innovation in a more comprehensive and in-depth way. 


\section{Research methods}

The research can be divided into the following four stages.

\subsection{The first stage: traditional DEA}

Referring to the related literatures on R\&D input-output efficiency of high-tech industry, this paper selects the inputoriented variable-scale BCC model to carry out the first phase of calculation. The main reason for choosing BCC model is its variable scale. Problems such as unequal competition and resource constraints in domestic high-tech industries may cause some decision-making units (DMU) to be unable to operate at the optimal scale, and the measurement of technical efficiency will be affected by scale efficiency. Considering this situation, BCC model adds convex conditions, allowing technical efficiency (TE) to be further decomposed into pure technical efficiency (PTE) and scale efficiency (SE), which is helpful to analyze the influencing factors of R\&D input-output efficiency in China's high-tech industry in recent years.

\subsection{The second stage: similar SFA}

On the basis of the previous research stage, the difference between the actual input and the target input of the final accounting unit is calculated, i.e. the input slack, and a similar SFA model is constructed with the input slack as the dependent variable and the circumstantial factor as the independent variable, so as to separate the efficiency values affected by the external environment and random error factors, and thus obtain the redundancy of the input of the decision-making unit caused only by the inefficiency of management.

\subsection{The third stage: adjusted DEA and malmquist index}

According to the method proposed by Fried ${ }^{[15]}$, the adjusted input data are substituted into BCC model again for efficiency analysis, thus obtaining the efficiency values of DMU after eliminating circumstantial factors and random factors. Compared with the traditional DEA model, the efficiency value thus obtained is closer to the real value. Since the data of DMU being evaluated is panel data including multiple time points, Malmquist TFP index can be used to analyze the effects of productivity changes, technical efficiency, and technological progress on productivity changes, respectively.

\subsection{The fourth stage: chain multiple mediation effect}

The total factor productivity excluding environmental and random factors is taken as the proxy variable to measure the innovation level in each region. The number of new ventures in high-tech industries, regional economic growth, and entrepreneurship in each region are included in the research framework, and an intermediary chain is formed according to the sequential characteristics of the intermediary variables. Through the intermediary effect model, the relationship between multiple variables and the process and mechanism of the influence between variables are analyzed simultaneously. This stage is mainly based on structural equation model (SEM) to carry out the above analysis. The following regression equations can be used to describe the relationship between variables:

$$
\begin{aligned}
& Y=c^{\prime} X+b_{1} M_{1}+b_{2} M_{2}+e_{1} \\
& M_{2}=a_{2} X+a_{3} M_{1}+e_{2} \\
& M_{1}=a_{1} X+e_{3}
\end{aligned}
$$

The corresponding path of chain multiple mediation effect is shown in Figure 1.

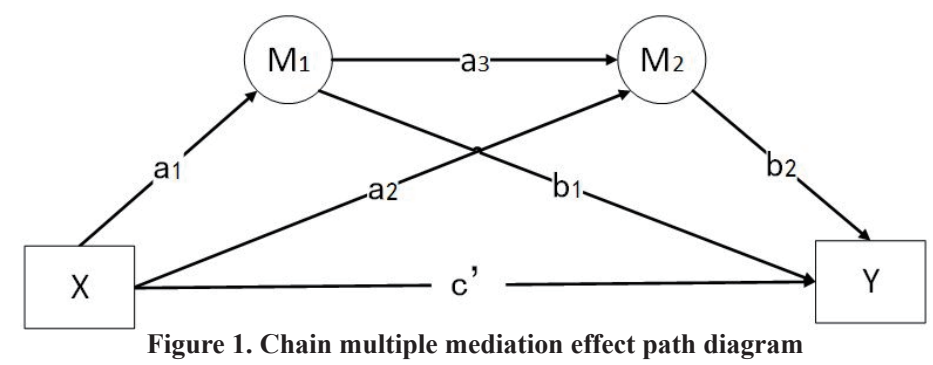

As shown in Figure 1, the multiple mediation model includes two mediation variables $M_{1}$ and $M_{2}$, at this time, the 
multiple mediation effect analysis can be carried out from three angles. The first is to analyze from the total mediation effect, namely: $a_{1} b_{1}+a_{2} b_{2}+a_{1} a_{3} b_{2}$. Secondly, on the premise of controlling other intermediate variables (such as control $M_{1}$ ), we can study the specific mediation effect of each intermediate variable, such as $a_{1} b_{1}, a_{2} b_{2}$ and $a_{1} a_{3} b_{2}$. Third, a comparative mediating effect can be obtained so as to be able to judge which of the effects of multiple mediating variables (e.g. $a_{1} b_{1}$, $a_{2} b_{2}$ ) is more effective, e.g $a_{1} a_{3} b_{2}-a_{2} b_{2}$, and $a_{1} b_{1}-a_{2} b_{2}, a_{1} a_{3} b_{2}-a_{1} b_{1}$ (Preacher and Hayes ${ }^{[16]}$ ).

\section{Variable selection and data description}

\subsection{Input-output indicators}

Data on capital input, personnel input and innovation output are all derived from China's High-tech Industry Statistical Yearbook. As capital investment data are often affected by price fluctuations, the relevant data should be reduced by using fixed asset investment price index (last year $=100$ ) of corresponding years in all provinces and cities, and the provincial fixed asset investment price index data of each year comes from China Statistical Yearbook. In addition, Tibet and Xinjiang were excluded from the sample due to the serious lack of data. In order to eliminate the time lag between input and output and the difference between innovation activity cycles, this study chooses the time lag as one year, i.e. the input data is 2004-2013 and the output data is 2005-2014. In addition, because the internal expenditure of R\&D funds, expenditure of new product development and expenditure of technological transformation not only have an impact on the input-output efficiency of high-tech industries in the current period, but also will have an important impact in some future periods, it is first necessary to convert the flow data into stock and then measure the impact of these three capital inputs on the innovation-output efficiency. Taking the calculation of R\&D fund stock as an example, the method of permanent storage is used to deal with it.

$$
x_{i, t}=(1-\delta) x_{i . t-1}+e_{i, t-1}
$$

In the $\mathrm{Eq}(2), x_{i, t}$ and $x_{i, t-1}$ are the $\mathrm{R} \& \mathrm{D}$ expenditures of the $t$ and $t-1$ years of the province $i$ respectively; $e_{i, t-1}$ is the R\&D expenditure of the $t-1$ year after eliminating the price factor; $\delta$ is the depreciation rate, according to Griliches ${ }^{[17]}$ can set it to $\delta=15 \%$ Assuming that the average annual growth rate of R\&D expenditures during the study period is $g$, the base $\mathrm{R} \& \mathrm{D}$ expenditure is

$$
x_{i, o}=e_{i, 0} /(g+\delta)
$$

$e_{i, 0}$ in the $\mathrm{Eq}(3)$ refers to the internal expenditure of $\mathrm{R} \& \mathrm{D}$ funds for the base period. On the basis of equations (2) and (3), the R\&D fund stock of each year can be obtained by using the perpetual inventory method. The above method can be used to process the funds for new product development and technological transformation, thus converting the flow data into stock. Input-output related variables are described in Table 1.

\begin{tabular}{|c|c|c|c|}
\hline Variable & Symbol & Unit & Definition \\
\hline Innovation Output 1 & y1 & Item & Number of patent applications \\
\hline Innovation Output 2 & y2 & Ten thousand yuan & Revenue from sales of new products \\
\hline $\begin{array}{l}\text { Innovation (personnel) } \\
\text { input } 1\end{array}$ & $\mathrm{x} 1$ & Person/Year & $\begin{array}{l}\text { Full-time equivalent of R\&D personnel refers to the sum of the } \\
\text { number of R\&D full-time personnel plus part-time personnel } \\
\text { converted into the current number of personnel according to } \\
\text { the workload during the reporting year }\end{array}$ \\
\hline $\begin{array}{l}\text { Innovation (capital) } \\
\text { investment } 2\end{array}$ & $x 2$ & Ten thousand yuan & $\begin{array}{l}\text { The stock of R\&D funds is calculated by the perpetual } \\
\text { inventory method. }\end{array}$ \\
\hline $\begin{array}{l}\text { Innovation (capital) } \\
\text { investment } 3\end{array}$ & $\mathrm{x} 3$ & Ten thousand yuan & $\begin{array}{l}\text { The stock of new product development funds shall be } \\
\text { accounted for by the perpetual inventory method. }\end{array}$ \\
\hline $\begin{array}{l}\text { Innovation (capital) } \\
\text { investment } 4\end{array}$ & $\mathrm{x} 4$ & Ten thousand yuan & $\begin{array}{l}\text { The stock of funds for technological transformation shall be } \\
\text { accounted for by the perpetual inventory method. }\end{array}$ \\
\hline
\end{tabular}

Table 1. Description of input-output variables

\subsection{Environmental variables}

Environmental variables are mainly used to reflect the geographic location of high-tech industries, macroenvironment, and government innovation support policies. Based on the relevant research foundation at home and abroad, 
and considering the characteristics of $R \& D$ and the availability of data, this paper mainly selects the following indicators:

\subsubsection{Regional real GDP}

Real GDP can accurately reflect regional differences and the real level of regional economic development, so this paper uses real GDP excluding price factors instead of nominal GDP. Generally speaking, the higher the regional economic development level, the more capable it is to invest in R\&D innovation in high-tech industries, and the higher the R\&D innovation level.

\subsubsection{Geographical location}

Whether a region is located in a superior geographical position is crucial to the development of high-tech industries in the region. This paper quantifies this variable by setting virtual variables, in which " 1 " is used to represent the eastern region, including Beijing, Tianjin, Hebei, Liaoning, Shanghai, Jiangsu, Zhejiang, Fujian, Shandong, Guangdong and Hainan. "2" means the central region, including Shanxi, Inner Mongolia, Jilin, Heilongjiang, Anhui, Jiangxi, Henan, Hubei and Hunan; "0" means the western region, including Chongqing, Sichuan, Guizhou, Yunnan, Shaanxi, Gansu, Qinghai, Guangxi and Ningxia.Due to the serious lack of data in Xinjiang and Tibet, this article will not consider it for the time being.

\subsubsection{Quality of local workers}

In view of the availability of data, this paper chooses the number of college students in the studied area as the characterization variable of laborer's quality. The higher the quality of local laborers, the more likely it is to provide more talents and intellectual support for the development of high-tech industries, and the more conducive to the improvement of R\&D efficiency.

\subsubsection{Local government innovation support}

Local government support has an important impact on the R\&D and innovation process of high-tech industries. This paper selects the government funds from the funds raised for scientific and technological activities to represent the innovation support of local governments. Generally speaking, local government support can provide guarantee for the infrastructure construction of high-tech industries in the region, as well as financial support. The greater the support, the more conducive to R\&D and innovation in high-tech industries.

\subsubsection{Regional science and technology development level}

Due to the spatial aggregation effect and spillover effect of high-tech industries, the degree of technological development in a region often plays an important role in the development of high-tech industries. This paper chooses the number of high-tech industrial enterprises and the number of scientific and technological institutions to represent the degree of scientific and technological development in a region. In general, the more high-tech enterprises and scientific and technological institutions there are, the more conducive they are to R\&D and innovation in high-tech industries.

Among the above environmental variables, except that the actual GDP of each region comes from China Statistical Yearbook, other data come from China High-tech Industry Statistical Yearbook. Descriptive statistics of relevant variables are shown in Table 2.

Table 2. Descriptive statistics of environmental variables

\begin{tabular}{|c|c|c|c|c|c|c|}
\hline Variable & Symbol & observed value & Mean value & Standard deviation & Minimum value & Maximum value \\
\hline Regional real GDP & $k_{1}$ & 261 & 13824.85 & 11858 & 572.37 & 62924.62 \\
\hline Geographical position & $k_{2}$ & 261 & 1 & 0.7894 & 0 & 2 \\
\hline Quality of local laborers & $k_{3}$ & 261 & 746585 & 437384.5 & 35983 & 1796665 \\
\hline $\begin{array}{l}\text { Local government innovation } \\
\text { support }\end{array}$ & $k_{4}$ & 261 & 36197.87 & 51416.87 & 10 & 289006.9 \\
\hline $\begin{array}{l}\text { Number of high-tech industrial } \\
\text { enterprises }\end{array}$ & $k_{5}$ & 261 & 853.29 & 1208.19 & 14 & 5874 \\
\hline $\begin{array}{c}\text { Number of scientific and } \\
\text { technological institutions }\end{array}$ & $k_{6}$ & 261 & 156.69 & 319.04 & 1 & 2474 \\
\hline
\end{tabular}

\subsection{Mediating effect variables}

Taking the total factor productivity (X) obtained by Malmquist index decomposition in the third stage as the independent variable. The dependent variable is the logarithm (Y) of the number of newly-built entrepreneurs in individual and private enterprises in high-tech industries. The variable is obtained by using the difference between the number of private enterprises and individual workers in urban employment at the end of the year in China Statistical Yearbook 2005- 
2014. The intermediate variables are respectively the logarithm of the number of new ventures (M1) and the logarithm of the actual GDP of each region (M2). Among them, the number of new ventures is obtained by year-on-year difference using the number of enterprises in the China Statistical Yearbook from 2005 to 2014. The actual GDP of each region comes from the China Statistical Yearbook.

\section{Empirical analysis}

\subsection{Empirical analysis of traditional DEA in the first stage}

DEAP software is used to preliminarily measure the comprehensive technical efficiency, pure technical efficiency and scale efficiency of 29 provinces and cities in China from 2005 to 2014. The results are shown in Tables 3, 4 and 5. According to the calculation results before adjustment, the average value of China's R\&D comprehensive technical efficiency is $0.48-0.67$ from 2005 to 2014, without considering the interference of external environment and random influence factors, which generally shows a rising trend. The average value of pure technical efficiency is $0.63-0.73$, with room for improvement to varying degrees. The average value of scale efficiency is $0.753-0.926$, and the overall efficiency level is relatively high. Generally speaking, the R\&D efficiency of most provinces is below the efficiency frontier. As the result does not eliminate the influence of circumstantial factors and random interference factors, it cannot accurately measure the true level of the comprehensive technical efficiency of each province, so further adjustment and measurement are needed.

Table 3. Technical efficiency estimation results of technological innovation

\begin{tabular}{|c|c|c|c|c|c|c|c|c|c|c|c|}
\hline \multicolumn{2}{|c|}{ Year } & 2005 & 2006 & 2007 & 2008 & 2009 & 2010 & 2011 & 2012 & 2013 & 2014 \\
\hline \multirow{2}{*}{ Beijing } & BA & 0.27 & 0.30 & 1.00 & 1.00 & 1.00 & 1.00 & 1.00 & 1.00 & 1.00 & 1.00 \\
\hline & AA & 0.45 & 0.45 & 1.00 & 1.00 & 1.00 & 1.00 & 1.00 & 1.00 & 0.97 & 0.92 \\
\hline \multirow{2}{*}{ Tianjin } & BA & 1.00 & 1.00 & 1.00 & 1.00 & 1.00 & 1.00 & 1.00 & 1.00 & 1.00 & 1.00 \\
\hline & AA & 1.00 & 1.00 & 0.90 & 0.83 & 0.82 & 0.92 & 0.64 & 0.87 & 1.00 & 0.92 \\
\hline \multirow{2}{*}{ Hebei } & BA & 0.12 & 0.09 & 0.10 & 0.40 & 0.28 & 0.43 & 0.28 & 0.44 & 0.43 & 0.50 \\
\hline & AA & 0.06 & 0.05 & 0.07 & 0.11 & 0.13 & 0.13 & 0.16 & 0.23 & 0.31 & 0.43 \\
\hline \multirow{2}{*}{ Shanxi } & BA & 1.00 & 1.00 & 1.00 & 1.00 & 1.00 & 1.00 & 0.83 & 0.95 & 0.87 & 0.52 \\
\hline & AA & 0.01 & 0.02 & 0.05 & 0.04 & 0.04 & 0.08 & 0.08 & 0.13 & 0.17 & 0.12 \\
\hline \multirow{2}{*}{ Inner Mongolia } & BA & 1.00 & 0.41 & 0.05 & 0.27 & 1.00 & 0.61 & 0.56 & 0.57 & 0.56 & 0.34 \\
\hline & AA & 0.01 & 0.00 & 0.00 & 0.00 & 0.02 & 0.02 & 0.02 & 0.02 & 0.03 & 0.03 \\
\hline \multirow{2}{*}{ Liaoning } & BA & 0.24 & 0.14 & 0.22 & 0.48 & 0.62 & 0.47 & 0.61 & 0.45 & 0.63 & 0.59 \\
\hline & AA & 0.22 & 0.16 & 0.24 & 0.36 & 0.32 & 0.30 & 0.40 & 0.43 & 0.49 & 0.49 \\
\hline \multirow{2}{*}{ Jilin } & BA & 0.42 & 0.12 & 0.19 & 0.58 & 0.65 & 0.35 & 0.56 & 0.64 & 0.61 & 0.54 \\
\hline & AA & 0.06 & 0.03 & 0.05 & 0.06 & 0.12 & 0.05 & 0.11 & 0.18 & 0.25 & 0.21 \\
\hline \multirow{2}{*}{ Heilongjiang } & BA & 0.50 & 0.06 & 0.08 & 0.23 & 0.19 & 0.21 & 0.24 & 0.30 & 0.29 & 0.45 \\
\hline & AA & 0.13 & 0.04 & 0.05 & 0.07 & 0.09 & 0.10 & 0.17 & 0.20 & 0.25 & 0.32 \\
\hline \multirow{2}{*}{ Shanghai } & BA & 0.86 & 0.53 & 0.63 & 0.88 & 0.50 & 0.55 & 0.73 & 0.57 & 0.70 & 0.87 \\
\hline & AA & 1.00 & 1.00 & 0.94 & 1.00 & 0.84 & 0.81 & 0.82 & 0.70 & 0.85 & 0.83 \\
\hline \multirow{2}{*}{ Jiangsu } & BA & 0.28 & 0.27 & 0.37 & 0.70 & 0.80 & 0.69 & 0.97 & 1.00 & 0.66 & 0.81 \\
\hline & AA & 0.61 & 0.69 & 0.94 & 1.00 & 1.00 & 1.00 & 1.00 & 1.00 & 1.00 & 1.00 \\
\hline \multirow{2}{*}{ Zhejiang } & BA & 0.18 & 0.14 & 0.17 & 0.43 & 0.60 & 0.46 & 0.50 & 0.63 & 0.71 & 0.58 \\
\hline & AA & 0.33 & 0.40 & 0.46 & 0.49 & 0.64 & 0.57 & 0.72 & 0.91 & 1.00 & 0.91 \\
\hline \multirow{2}{*}{ Anhui } & BA & 0.74 & 0.50 & 0.21 & 0.34 & 0.76 & 1.00 & 1.00 & 1.00 & 1.00 & 1.00 \\
\hline & AA & 0.05 & 0.06 & 0.06 & 0.07 & 0.23 & 0.33 & 0.64 & 0.83 & 1.00 & 1.00 \\
\hline \multirow{2}{*}{ Fujian } & BA & 0.86 & 0.68 & 0.66 & 0.86 & 0.82 & 0.77 & 1.00 & 0.81 & 0.56 & 0.51 \\
\hline & AA & 0.72 & 0.71 & 0.72 & 0.68 & 0.69 & 0.82 & 0.63 & 0.70 & 0.69 & 0.66 \\
\hline \multirow{2}{*}{ Jiangxi } & BA & 0.15 & 0.11 & 0.10 & 0.18 & 0.29 & 0.37 & 0.28 & 0.42 & 0.53 & 0.69 \\
\hline & AA & 0.09 & 0.07 & 0.08 & 0.08 & 0.14 & 0.18 & 0.17 & 0.26 & 0.42 & 0.58 \\
\hline \multirow{2}{*}{ Shandong } & BA & 0.45 & 0.24 & 0.38 & 0.50 & 0.54 & 0.63 & 0.65 & 0.60 & 0.53 & 0.58 \\
\hline & AA & 0.51 & 0.54 & 0.67 & 0.71 & 0.84 & 0.84 & 0.85 & 0.80 & 0.76 & 0.75 \\
\hline \multirow{2}{*}{ Henan } & BA & 0.15 & 0.12 & 0.17 & 0.69 & 0.51 & 0.71 & 0.48 & 0.51 & 1.00 & 1.00 \\
\hline & AA & 0.08 & 0.09 & 0.14 & 0.20 & 0.30 & 0.33 & 0.44 & 0.51 & 1.00 & 1.00 \\
\hline \multirow{2}{*}{ Hubei } & BA & 0.16 & 0.13 & 0.16 & 0.36 & 0.54 & 0.50 & 0.54 & 0.52 & 0.55 & 0.52 \\
\hline & AA & 0.16 & 0.11 & 0.17 & 0.18 & 0.38 & 0.37 & 0.44 & 0.58 & 0.68 & 0.58 \\
\hline \multirow{2}{*}{ Hunan } & BA & 0.19 & 0.15 & 0.12 & 0.70 & 0.94 & 0.86 & 1.00 & 1.00 & 1.00 & 0.85 \\
\hline & $\mathrm{AA}$ & 0.04 & 0.04 & 0.05 & 0.11 & 0.27 & 0.26 & 0.56 & 0.59 & 0.79 & 0.86 \\
\hline
\end{tabular}




\begin{tabular}{|c|c|c|c|c|c|c|c|c|c|c|c|}
\hline \multirow{2}{*}{ Guangdong } & $\mathrm{BA}$ & 0.35 & 0.33 & 0.32 & 1.00 & 0.83 & 1.00 & 0.91 & 0.95 & 1.00 & 1.00 \\
\hline & AA & 1.00 & 1.00 & 1.00 & 1.00 & 1.00 & 1.00 & 1.00 & 1.00 & 1.00 & 1.00 \\
\hline \multirow{2}{*}{ Guangxi } & BA & 0.56 & 0.15 & 0.11 & 0.55 & 0.49 & 0.41 & 0.52 & 0.53 & 0.68 & 0.57 \\
\hline & AA & 0.04 & 0.02 & 0.03 & 0.03 & 0.05 & 0.04 & 0.08 & 0.11 & 0.17 & 0.16 \\
\hline \multirow{2}{*}{ Hainan } & BA & 1.00 & 1.00 & 1.00 & 1.00 & 1.00 & 1.00 & 1.00 & 1.00 & 1.00 & 0.85 \\
\hline & AA & 0.00 & 0.00 & 0.01 & 0.00 & 0.05 & 0.02 & 0.07 & 0.12 & 0.14 & 0.15 \\
\hline \multirow{2}{*}{ Chongqing } & BA & 0.29 & 0.25 & 0.17 & 0.44 & 0.94 & 0.71 & 1.00 & 0.81 & 0.94 & 0.97 \\
\hline & AA & 0.07 & 0.08 & 0.08 & 0.13 & 0.23 & 0.22 & 0.41 & 0.34 & 0.51 & 0.53 \\
\hline \multirow{2}{*}{ Sichuan } & $\mathrm{BA}$ & 0.21 & 0.23 & 0.25 & 0.29 & 0.54 & 0.35 & 1.00 & 0.82 & 0.70 & 0.98 \\
\hline & AA & 0.29 & 0.33 & 0.44 & 0.38 & 0.53 & 0.41 & 0.55 & 0.80 & 0.84 & 0.90 \\
\hline \multirow{2}{*}{ Guizhou } & $\mathrm{BA}$ & 0.22 & 0.16 & 0.13 & 0.49 & 0.58 & 0.62 & 0.42 & 0.43 & 0.42 & 0.45 \\
\hline & AA & 0.05 & 0.06 & 0.10 & 0.11 & 0.14 & 0.18 & 0.18 & 0.28 & 0.35 & 0.37 \\
\hline \multirow{2}{*}{ Yunnan } & BA & 1.00 & 0.20 & 0.46 & 1.00 & 1.00 & 0.80 & 0.92 & 0.81 & 0.76 & 0.64 \\
\hline & AA & 0.08 & 0.02 & 0.08 & 0.03 & 0.07 & 0.04 & 0.09 & 0.14 & 0.13 & 0.14 \\
\hline \multirow{2}{*}{ Shaanxi } & BA & 0.09 & 0.07 & 0.08 & 0.17 & 0.21 & 0.23 & 0.27 & 0.25 & 0.29 & 0.24 \\
\hline & $\mathrm{AA}$ & 0.16 & 0.15 & 0.21 & 0.20 & 0.19 & 0.22 & 0.29 & 0.32 & 0.44 & 0.37 \\
\hline \multirow{2}{*}{ Gansu } & BA & 0.53 & 0.27 & 0.17 & 0.48 & 0.42 & 0.94 & 0.46 & 0.68 & 0.59 & 0.63 \\
\hline & AA & 0.02 & 0.01 & 0.01 & 0.02 & 0.03 & 0.05 & 0.05 & 0.08 & 0.08 & 0.11 \\
\hline \multirow{2}{*}{ Qinghai } & BA & 1.00 & 1.00 & 1.00 & 0.39 & 0.59 & 1.00 & 1.00 & 0.16 & 0.99 & 0.49 \\
\hline & AA & 0.00 & 0.01 & 0.01 & 0.00 & 0.01 & 0.00 & 0.01 & 0.00 & 0.01 & 0.00 \\
\hline \multirow{2}{*}{ Ningxia } & BA & 0.13 & 0.24 & 0.10 & 0.28 & 0.31 & 0.41 & 0.69 & 0.69 & 1.00 & 0.35 \\
\hline & AA & 0.01 & 0.01 & 0.01 & 0.02 & 0.02 & 0.03 & 0.04 & 0.04 & 0.08 & 0.03 \\
\hline \multirow{2}{*}{ Average value } & BA & 0.48 & 0.34 & 0.36 & 0.58 & 0.65 & 0.66 & 0.70 & 0.67 & 0.72 & 0.67 \\
\hline & $\mathrm{AA}$ & 0.25 & 0.25 & 0.30 & 0.31 & 0.35 & 0.36 & 0.40 & 0.45 & 0.53 & 0.53 \\
\hline
\end{tabular}

Note: "BA" means Before Adjustment, "AA" means After Adjustment

Table 4. Pure technical efficiency estimation results of technological innovation

\begin{tabular}{|c|c|c|c|c|c|c|c|c|c|c|c|}
\hline \multicolumn{2}{|c|}{$\begin{array}{r}\text { Year } \\
\text { Provinces }\end{array}$} & 2005 & 2006 & 2007 & 2008 & 2009 & 2010 & 2011 & 2012 & 2013 & 2014 \\
\hline \multirow{2}{*}{ Beijing } & $\mathrm{BA}$ & 0.50 & 0.59 & 1.00 & 1.00 & 1.00 & 1.00 & 1.00 & 1.00 & 1.00 & 1.00 \\
\hline & $\mathrm{AA}$ & 0.96 & 0.96 & 1.00 & 1.00 & 1.00 & 1.00 & 1.00 & 1.00 & 1.00 & 1.00 \\
\hline \multirow{2}{*}{ Tianjin } & $\mathrm{BA}$ & 1.00 & 1.00 & 1.00 & 1.00 & 1.00 & 1.00 & 1.00 & 1.00 & 1.00 & 1.00 \\
\hline & AA & 1.00 & 1.00 & 1.00 & 1.00 & 1.00 & 1.00 & 1.00 & 1.00 & 1.00 & 1.00 \\
\hline \multirow{2}{*}{ Hebei } & BA & 0.13 & 0.28 & 0.15 & 0.44 & 0.34 & 0.48 & 0.28 & 0.45 & 0.44 & 0.50 \\
\hline & $\mathrm{AA}$ & 1.00 & 1.00 & 1.00 & 1.00 & 1.00 & 1.00 & 1.00 & 1.00 & 0.99 & 1.00 \\
\hline \multirow{2}{*}{ Shanxi } & $\mathrm{BA}$ & 1.00 & 1.00 & 1.00 & 1.00 & 1.00 & 1.00 & 0.85 & 0.98 & 0.88 & 0.55 \\
\hline & AA & 0.99 & 0.99 & 0.99 & 1.00 & 1.00 & 1.00 & 1.00 & 1.00 & 1.00 & 1.00 \\
\hline \multirow{2}{*}{ Inner Mongolia } & BA & 1.00 & 0.49 & 0.15 & 0.59 & 1.00 & 0.78 & 0.61 & 0.71 & 0.62 & 0.49 \\
\hline & AA & 0.98 & 0.98 & 0.98 & 0.99 & 1.00 & 1.00 & 1.00 & 1.00 & 1.00 & 1.00 \\
\hline \multirow{2}{*}{ Liaoning } & BA & 0.59 & 0.29 & 0.27 & 0.48 & 0.75 & 0.53 & 0.61 & 0.46 & 0.66 & 0.60 \\
\hline & AA & 1.00 & 0.99 & 1.00 & 1.00 & 1.00 & 1.00 & 1.00 & 0.99 & 1.00 & 0.95 \\
\hline \multirow{2}{*}{ Jilin } & BA & 0.44 & 0.32 & 0.40 & 0.59 & 0.67 & 0.35 & 0.56 & 0.65 & 0.63 & 0.57 \\
\hline & AA & 0.99 & 0.98 & 0.97 & 0.97 & 0.97 & 0.97 & 0.97 & 0.97 & 0.98 & 0.96 \\
\hline \multirow{2}{*}{ Heilongjiang } & $\mathrm{BA}$ & 0.64 & 0.22 & 0.10 & 0.25 & 0.21 & 0.27 & 0.27 & 0.31 & 0.32 & 0.46 \\
\hline & AA & 0.98 & 0.98 & 0.99 & 1.00 & 0.97 & 0.96 & 0.94 & 0.95 & 0.95 & 0.96 \\
\hline \multirow{2}{*}{ Shanghai } & $\mathrm{BA}$ & 1.00 & 1.00 & 0.91 & 1.00 & 0.73 & 0.70 & 0.77 & 0.57 & 0.75 & 0.88 \\
\hline & $\mathrm{AA}$ & 1.00 & 1.00 & 0.99 & 1.00 & 0.90 & 0.89 & 0.96 & 0.89 & 0.93 & 0.99 \\
\hline \multirow{2}{*}{ Jiangsu } & BA & 0.60 & 0.36 & 0.78 & 1.00 & 1.00 & 1.00 & 1.00 & 1.00 & 1.00 & 1.00 \\
\hline & AA & 0.98 & 0.87 & 0.98 & 1.00 & 1.00 & 1.00 & 1.00 & 1.00 & 1.00 & 1.00 \\
\hline \multirow{2}{*}{ Zhejiang } & BA & 0.38 & 0.38 & 0.55 & 0.57 & 0.83 & 0.63 & 0.74 & 0.90 & 1.00 & 0.88 \\
\hline & AA & 0.91 & 0.85 & 0.81 & 0.79 & 0.91 & 0.80 & 0.85 & 0.92 & 1.00 & 0.91 \\
\hline \multirow{2}{*}{ Anhui } & BA & 0.91 & 0.51 & 0.28 & 0.64 & 1.00 & 1.00 & 1.00 & 1.00 & 1.00 & 1.00 \\
\hline & AA & 0.98 & 0.99 & 0.99 & 1.00 & 1.00 & 1.00 & 1.00 & 1.00 & 1.00 & 1.00 \\
\hline \multirow{2}{*}{ Fujian } & BA & 0.88 & 0.68 & 0.78 & 0.93 & 0.91 & 0.89 & 1.00 & 0.81 & 0.57 & 0.52 \\
\hline & AA & 1.00 & 0.98 & 0.98 & 1.00 & 0.99 & 0.99 & 1.00 & 0.94 & 0.81 & 0.77 \\
\hline \multirow{2}{*}{ Jiangxi } & BA & 0.23 & 0.22 & 0.12 & 0.20 & 0.29 & 0.38 & 0.29 & 0.42 & 0.53 & 0.70 \\
\hline & AA & 0.97 & 0.99 & 0.99 & 0.99 & 0.96 & 0.95 & 0.93 & 0.90 & 0.91 & 0.98 \\
\hline \multirow{2}{*}{ Shandong } & BA & 0.87 & 0.58 & 0.73 & 0.65 & 0.65 & 0.78 & 0.72 & 0.69 & 0.66 & 0.66 \\
\hline & AA & 1.00 & 1.00 & 1.00 & 0.95 & 0.95 & 0.99 & 0.94 & 0.87 & 0.84 & 0.78 \\
\hline \multirow{2}{*}{ Henan } & BA & 0.15 & 0.66 & 0.45 & 0.79 & 0.79 & 0.84 & 0.53 & 0.56 & 1.00 & 1.00 \\
\hline & AA & 0.97 & 0.98 & 1.00 & 1.00 & 1.00 & 1.00 & 0.97 & 0.96 & 1.00 & 1.00 \\
\hline
\end{tabular}




\begin{tabular}{|c|c|c|c|c|c|c|c|c|c|c|c|}
\hline \multirow{2}{*}{ Hubei } & $\mathrm{BA}$ & 0.84 & 0.51 & 0.34 & 0.44 & 0.62 & 0.53 & 0.54 & 0.55 & 0.60 & 0.52 \\
\hline & $\mathrm{AA}$ & 1.00 & 1.00 & 1.00 & 1.00 & 1.00 & 1.00 & 1.00 & 1.00 & 0.99 & 0.93 \\
\hline \multirow{2}{*}{ Hunan } & BA & 0.29 & 0.18 & 0.17 & 0.81 & 1.00 & 0.94 & 1.00 & 1.00 & 1.00 & 0.85 \\
\hline & AA & 1.00 & 1.00 & 1.00 & 1.00 & 1.00 & 1.00 & 1.00 & 1.00 & 1.00 & 0.97 \\
\hline \multirow{2}{*}{ Guangdong } & BA & 1.00 & 1.00 & 1.00 & 1.00 & 1.00 & 1.00 & 1.00 & 1.00 & 1.00 & 1.00 \\
\hline & AA & 1.00 & 1.00 & 1.00 & 1.00 & 1.00 & 1.00 & 1.00 & 1.00 & 1.00 & 1.00 \\
\hline \multirow{2}{*}{ Guangxi } & $\mathrm{BA}$ & 0.74 & 0.23 & 0.16 & 0.56 & 0.50 & 0.42 & 0.54 & 0.55 & 0.70 & 0.61 \\
\hline & $\mathrm{AA}$ & 0.97 & 0.97 & 0.96 & 0.97 & 0.99 & 0.98 & 0.98 & 0.99 & 1.00 & 0.98 \\
\hline \multirow{2}{*}{ Hainan } & BA & 1.00 & 1.00 & 1.00 & 1.00 & 1.00 & 1.00 & 1.00 & 1.00 & 1.00 & 0.88 \\
\hline & $\mathrm{AA}$ & 1.00 & 1.00 & 1.00 & 1.00 & 1.00 & 1.00 & 1.00 & 1.00 & 1.00 & 1.00 \\
\hline \multirow{2}{*}{ Chongqing } & BA & 0.31 & 0.49 & 0.27 & 0.61 & 0.96 & 0.77 & 1.00 & 0.81 & 0.94 & 0.98 \\
\hline & $\mathrm{AA}$ & 0.95 & 0.94 & 0.94 & 0.95 & 0.98 & 0.98 & 1.00 & 0.97 & 0.99 & 1.00 \\
\hline \multirow{2}{*}{ Sichuan } & BA & 0.26 & 0.34 & 0.54 & 0.44 & 0.56 & 0.52 & 1.00 & 0.82 & 0.74 & 1.00 \\
\hline & $\mathrm{AA}$ & 0.96 & 0.95 & 0.95 & 0.90 & 0.90 & 0.92 & 1.00 & 0.98 & 0.96 & 1.00 \\
\hline \multirow{2}{*}{ Guizhou } & BA & 0.25 & 0.73 & 0.39 & 0.71 & 0.69 & 0.67 & 0.45 & 0.43 & 0.42 & 0.46 \\
\hline & AA & 0.93 & 0.94 & 0.95 & 0.94 & 0.96 & 0.96 & 0.94 & 0.91 & 0.90 & 0.90 \\
\hline \multirow{2}{*}{ Yunnan } & BA & 1.00 & 0.55 & 1.00 & 1.00 & 1.00 & 0.84 & 0.94 & 0.81 & 0.77 & 0.66 \\
\hline & $\mathrm{AA}$ & 1.00 & 0.98 & 0.99 & 0.97 & 1.00 & 1.00 & 0.99 & 0.99 & 1.00 & 0.99 \\
\hline \multirow{2}{*}{ Shaanxi } & BA & 0.17 & 0.12 & 0.16 & 0.21 & 0.22 & 0.28 & 0.28 & 0.25 & 0.32 & 0.24 \\
\hline & $\mathrm{AA}$ & 0.92 & 0.89 & 0.93 & 0.88 & 0.85 & 0.84 & 0.85 & 0.78 & 0.80 & 0.77 \\
\hline \multirow{2}{*}{ Gansu } & BA & 1.00 & 0.27 & 0.18 & 0.50 & 0.46 & 0.95 & 0.46 & 0.74 & 0.64 & 0.69 \\
\hline & AA & 0.95 & 0.98 & 0.97 & 0.98 & 0.99 & 0.99 & 0.99 & 1.00 & 1.00 & 1.00 \\
\hline \multirow{2}{*}{ Qinghai } & BA & 1.00 & 1.00 & 1.00 & 1.00 & 1.00 & 1.00 & 1.00 & 1.00 & 1.00 & 1.00 \\
\hline & AA & 0.96 & 0.97 & 0.96 & 0.97 & 0.98 & 0.98 & 0.98 & 0.99 & 1.00 & 1.00 \\
\hline \multirow{2}{*}{ Ningxia } & BA & 0.13 & 0.24 & 0.11 & 0.32 & 0.49 & 0.47 & 0.73 & 0.83 & 1.00 & 0.49 \\
\hline & $\mathrm{AA}$ & 0.95 & 0.95 & 0.95 & 0.95 & 0.97 & 0.97 & 0.97 & 0.98 & 0.99 & 1.00 \\
\hline \multirow{2}{*}{ Average value } & $\mathrm{BA}$ & 0.63 & 0.53 & 0.52 & 0.68 & 0.75 & 0.73 & 0.73 & 0.73 & 0.77 & 0.73 \\
\hline & $\mathrm{AA}$ & 0.98 & 0.97 & 0.97 & 0.97 & 0.98 & 0.97 & 0.97 & 0.96 & 0.97 & 0.96 \\
\hline
\end{tabular}

Note: "BA" means Before Adjustment, "AA" means After Adjustment

Table 5. Estimate results of scale efficiency of technological innovation

\begin{tabular}{|c|c|c|c|c|c|c|c|c|c|c|c|}
\hline $\begin{array}{r}\text { Ye } \\
\text { Provin }\end{array}$ & & 2005 & 2006 & 2007 & 2008 & 2009 & 2010 & 2011 & 2012 & 2013 & 2014 \\
\hline \multirow{2}{*}{ Beijing } & BA & $\begin{array}{c}0.530 \\
\text { (drs) }\end{array}$ & $\begin{array}{c}0.510 \\
\text { (drs) }\end{array}$ & $\begin{array}{c}1.000 \\
(\mathrm{crs})\end{array}$ & $\begin{array}{c}1.000 \\
\text { (crs) }\end{array}$ & $\begin{array}{c}1.000 \\
(\mathrm{crs})\end{array}$ & $\begin{array}{c}1.000 \\
(\mathrm{crs})\end{array}$ & $\begin{array}{l}1.000 \\
\text { (crs) }\end{array}$ & $\begin{array}{c}1.000 \\
\text { (crs) }\end{array}$ & $\begin{array}{c}1.000 \\
(\mathrm{crs})\end{array}$ & $\begin{array}{c}1.000 \\
\text { (crs) }\end{array}$ \\
\hline & AA & $\begin{array}{c}0.463 \\
\text { (irs) }\end{array}$ & $\begin{array}{c}0.469 \\
\text { (irs) }\end{array}$ & $\begin{array}{l}1.000 \\
\text { (crs) }\end{array}$ & $\begin{array}{l}1.000 \\
\text { (crs) }\end{array}$ & $\begin{array}{l}1.000 \\
\text { (crs) }\end{array}$ & $\begin{array}{l}1.000 \\
\text { (crs) }\end{array}$ & $\begin{array}{l}1.000 \\
\text { (crs) }\end{array}$ & $\begin{array}{l}1.000 \\
(\mathrm{crs})\end{array}$ & $\begin{array}{c}0.974 \\
\text { (irs) }\end{array}$ & $\begin{array}{c}0.923 \\
\text { (irs) }\end{array}$ \\
\hline \multirow{2}{*}{ Tianjin } & BA & $\begin{array}{c}1.000 \\
(\mathrm{crs})\end{array}$ & $\begin{array}{l}1.000 \\
(\mathrm{crs})\end{array}$ & $\begin{array}{c}1.000 \\
(\mathrm{crs})\end{array}$ & $\begin{array}{c}1.000 \\
\text { (crs) }\end{array}$ & $\begin{array}{c}1.000 \\
\text { (crs) }\end{array}$ & $\begin{array}{c}1.000 \\
\text { (crs) }\end{array}$ & $\begin{array}{c}1.000 \\
\text { (crs) }\end{array}$ & $\begin{array}{c}1.000 \\
(\mathrm{crs})\end{array}$ & $\begin{array}{l}1.000 \\
(\mathrm{crs})\end{array}$ & $\begin{array}{c}1.000 \\
(\mathrm{crs})\end{array}$ \\
\hline & AA & $\begin{array}{l}1.000 \\
(\mathrm{crs})\end{array}$ & $\begin{array}{l}1.000 \\
(\mathrm{crs})\end{array}$ & $\begin{array}{c}0.900 \\
\text { (irs) }\end{array}$ & $\begin{array}{c}0.831 \\
\text { (irs) }\end{array}$ & $\begin{array}{c}0.824 \\
\text { (irs) }\end{array}$ & $\begin{array}{c}0.920 \\
\text { (irs) }\end{array}$ & $\begin{array}{c}0.637 \\
\text { (irs) }\end{array}$ & $\begin{array}{c}0.869 \\
\text { (irs) }\end{array}$ & $\begin{array}{l}1.000 \\
\text { (crs) }\end{array}$ & $\begin{array}{c}0.921 \\
\text { (irs) }\end{array}$ \\
\hline \multirow{2}{*}{ Hebei } & BA & $\begin{array}{c}0.944 \\
\text { (drs) }\end{array}$ & $\begin{array}{c}0.318 \\
\text { (drs) }\end{array}$ & $\begin{array}{c}0.672 \\
\text { (drs) }\end{array}$ & $\begin{array}{c}0.902 \\
\text { (drs) }\end{array}$ & $\begin{array}{c}0.820 \\
\text { (drs) }\end{array}$ & $\begin{array}{c}0.887 \\
\text { (drs) }\end{array}$ & $\begin{array}{c}0.988 \\
\text { (irs) }\end{array}$ & $\begin{array}{c}0.985 \\
\text { (irs) }\end{array}$ & $\begin{array}{c}0.989 \\
\text { (drs) }\end{array}$ & $\begin{array}{c}0.992 \\
\text { (irs) }\end{array}$ \\
\hline & AA & $\begin{array}{c}0.058 \\
\text { (irs) }\end{array}$ & $\begin{array}{c}0.054 \\
\text { (irs) }\end{array}$ & $\begin{array}{c}0.071 \\
\text { (irs) }\end{array}$ & $\begin{array}{c}0.107 \\
\text { (irs) }\end{array}$ & $\begin{array}{c}0.130 \\
\text { (irs) }\end{array}$ & $\begin{array}{c}0.128 \\
\text { (irs) }\end{array}$ & $\begin{array}{c}0.160 \\
\text { (irs) }\end{array}$ & $\begin{array}{c}0.227 \\
\text { (irs) }\end{array}$ & $\begin{array}{c}0.314 \\
\text { (irs) }\end{array}$ & $\begin{array}{c}0.427 \\
\text { (irs) }\end{array}$ \\
\hline \multirow{2}{*}{ Shanxi } & BA & $\begin{array}{l}1.000 \\
(\mathrm{crs})\end{array}$ & $\begin{array}{l}1.000 \\
\text { (crs) }\end{array}$ & $\begin{array}{l}1.000 \\
\text { (crs) }\end{array}$ & $\begin{array}{l}1.000 \\
\text { (crs) }\end{array}$ & $\begin{array}{l}1.000 \\
\text { (crs) }\end{array}$ & $\begin{array}{c}1.000 \\
\text { (crs) }\end{array}$ & $\begin{array}{c}0.980 \\
\text { (irs) }\end{array}$ & $\begin{array}{c}0.974 \\
\text { (irs) }\end{array}$ & $\begin{array}{c}0.994 \\
\text { (drs) }\end{array}$ & $\begin{array}{c}0.944 \\
\text { (irs) }\end{array}$ \\
\hline & AA & $\begin{array}{c}0.012 \\
\text { (irs) }\end{array}$ & $\begin{array}{c}0.022 \\
\text { (irs) }\end{array}$ & $\begin{array}{c}0.050 \\
\text { (irs) }\end{array}$ & $\begin{array}{c}0.036 \\
\text { (irs) }\end{array}$ & $\begin{array}{c}0.041 \\
\text { (irs) }\end{array}$ & $\begin{array}{c}0.081 \\
\text { (irs) }\end{array}$ & $\begin{array}{c}0.076 \\
\text { (irs) }\end{array}$ & $\begin{array}{c}0.127 \\
\text { (irs) }\end{array}$ & $\begin{array}{c}0.169 \\
\text { (irs) }\end{array}$ & $\begin{array}{c}0.118 \\
\text { (irs) }\end{array}$ \\
\hline \multirow{2}{*}{ Inner Mongolia } & BA & $\begin{array}{c}1.000 \\
\text { (crs) }\end{array}$ & $\begin{array}{c}0.838 \\
\text { (irs) }\end{array}$ & $\begin{array}{c}0.336 \\
\text { (irs) }\end{array}$ & $\begin{array}{c}0.451 \\
\text { (irs) }\end{array}$ & $\begin{array}{l}1.000 \\
\text { (crs) }\end{array}$ & $\begin{array}{c}0.781 \\
\text { (irs) }\end{array}$ & $\begin{array}{c}0.926 \\
\text { (irs) }\end{array}$ & $\begin{array}{c}0.793 \\
\text { (irs) }\end{array}$ & $\begin{array}{c}0.910 \\
\text { (irs) }\end{array}$ & $\begin{array}{c}0.691 \\
\text { (irs) }\end{array}$ \\
\hline & AA & $\begin{array}{c}0.007 \\
\text { (irs) }\end{array}$ & $\begin{array}{c}0.003 \\
\text { (irs) }\end{array}$ & $\begin{array}{c}0.002 \\
\text { (irs) }\end{array}$ & $\begin{array}{c}0.002 \\
\text { (irs) }\end{array}$ & $\begin{array}{c}0.023 \\
\text { (irs) }\end{array}$ & $\begin{array}{c}0.015 \\
\text { (irs) }\end{array}$ & $\begin{array}{c}0.017 \\
\text { (irs) }\end{array}$ & $\begin{array}{c}0.015 \\
\text { (irs) }\end{array}$ & $\begin{array}{c}0.026 \\
\text { (irs) }\end{array}$ & $\begin{array}{c}0.025 \\
\text { (irs) }\end{array}$ \\
\hline \multirow{2}{*}{ Liaoning } & BA & $\begin{array}{c}0.414 \\
\text { (drs) }\end{array}$ & $\begin{array}{c}0.500 \\
\text { (drs) }\end{array}$ & $\begin{array}{c}0.807 \\
\text { (drs) }\end{array}$ & $\begin{array}{c}0.989 \\
\text { (drs) }\end{array}$ & $\begin{array}{c}0.831 \\
\text { (drs) }\end{array}$ & $\begin{array}{c}0.880 \\
(\mathrm{drs})\end{array}$ & $\begin{array}{c}0.999 \\
\text { (irs) }\end{array}$ & $\begin{array}{c}0.994 \\
\text { (irs) }\end{array}$ & $\begin{array}{c}0.951 \\
(\mathrm{drs})\end{array}$ & $\begin{array}{c}0.991 \\
\text { (irs) }\end{array}$ \\
\hline & AA & $\begin{array}{c}0.218 \\
\text { (irs) }\end{array}$ & $\begin{array}{c}0.162 \\
\text { (irs) }\end{array}$ & $\begin{array}{c}0.239 \\
\text { (irs) }\end{array}$ & $\begin{array}{c}0.356 \\
\text { (irs) }\end{array}$ & $\begin{array}{c}0.319 \\
\text { (irs) }\end{array}$ & $\begin{array}{c}0.303 \\
\text { (irs) }\end{array}$ & $\begin{array}{c}0.399 \\
\text { (irs) }\end{array}$ & $\begin{array}{c}0.435 \\
\text { (irs) }\end{array}$ & $\begin{array}{c}0.490 \\
\text { (irs) }\end{array}$ & $\begin{array}{c}0.510 \\
\text { (irs) }\end{array}$ \\
\hline \multirow{2}{*}{ Jilin } & BA & $\begin{array}{c}0.949 \\
\text { (drs) }\end{array}$ & $\begin{array}{c}0.388 \\
\text { (drs) }\end{array}$ & $\begin{array}{c}0.468 \\
\text { (drs) }\end{array}$ & $\begin{array}{c}0.980 \\
\text { (drs) }\end{array}$ & $\begin{array}{c}0.969 \\
\text { (drs) }\end{array}$ & $\begin{array}{c}0.989 \\
\text { (drs) }\end{array}$ & $\begin{array}{c}0.989 \\
\text { (irs) }\end{array}$ & $\begin{array}{c}0.979 \\
\text { (irs) }\end{array}$ & $\begin{array}{c}0.962 \\
\text { (irs) }\end{array}$ & $\begin{array}{c}0.959 \\
\text { (irs) }\end{array}$ \\
\hline & AA & $\begin{array}{c}0.062 \\
\text { (irs) }\end{array}$ & $\begin{array}{c}0.029 \\
\text { (irs) }\end{array}$ & $\begin{array}{c}0.052 \\
\text { (irs) }\end{array}$ & $\begin{array}{c}0.059 \\
\text { (irs) }\end{array}$ & $\begin{array}{c}0.118 \\
\text { (irs) }\end{array}$ & $\begin{array}{c}0.054 \\
\text { (irs) }\end{array}$ & $\begin{array}{c}0.113 \\
\text { (irs) }\end{array}$ & $\begin{array}{c}0.181 \\
\text { (irs) }\end{array}$ & $\begin{array}{c}0.257 \\
\text { (irs) }\end{array}$ & $\begin{array}{c}0.219 \\
\text { (irs) }\end{array}$ \\
\hline \multirow{2}{*}{ Heilongjiang } & BA & $\begin{array}{c}0.774 \\
\text { (drs) }\end{array}$ & $\begin{array}{c}0.272 \\
\text { (drs) }\end{array}$ & $\begin{array}{c}0.800 \\
\text { (drs) }\end{array}$ & $\begin{array}{c}0.912 \\
\text { (drs) }\end{array}$ & $\begin{array}{c}0.887 \\
\text { (drs) }\end{array}$ & $\begin{array}{c}0.762 \\
\text { (drs) }\end{array}$ & $\begin{array}{c}0.874 \\
\text { (drs) }\end{array}$ & $\begin{array}{c}0.990 \\
\text { (irs) }\end{array}$ & $\begin{array}{l}0.911 \\
\text { (drs) }\end{array}$ & $\begin{array}{c}0.978 \\
\text { (irs) }\end{array}$ \\
\hline & AA & $\begin{array}{c}0.133 \\
\text { (irs) }\end{array}$ & $\begin{array}{c}0.036 \\
\text { (irs) }\end{array}$ & $\begin{array}{c}0.054 \\
\text { (irs) }\end{array}$ & $\begin{array}{c}0.072 \\
\text { (irs) }\end{array}$ & $\begin{array}{c}0.095 \\
\text { (irs) }\end{array}$ & $\begin{array}{c}0.105 \\
\text { (irs) }\end{array}$ & $\begin{array}{c}0.176 \\
\text { (irs) }\end{array}$ & $\begin{array}{c}0.211 \\
\text { (irs) }\end{array}$ & $\begin{array}{c}0.259 \\
\text { (irs) }\end{array}$ & $\begin{array}{c}0.338 \\
\text { (irs) }\end{array}$ \\
\hline
\end{tabular}




\begin{tabular}{|c|c|c|c|c|c|c|c|c|c|c|c|}
\hline \multirow{2}{*}{ Shanghai } & BA & $\begin{array}{c}0.862 \\
\text { (drs) }\end{array}$ & $\begin{array}{c}0.528 \\
\text { (drs) }\end{array}$ & $\begin{array}{c}0.690 \\
\text { (drs) }\end{array}$ & $\begin{array}{c}0.875 \\
(\mathrm{drs})\end{array}$ & $\begin{array}{c}0.690 \\
\text { (drs) }\end{array}$ & $\begin{array}{c}0.783 \\
\text { (drs) }\end{array}$ & $\begin{array}{c}0.958 \\
\text { (drs) }\end{array}$ & $\begin{array}{c}0.998 \\
\text { (irs) }\end{array}$ & $\begin{array}{c}0.928 \\
\text { (drs) }\end{array}$ & $\begin{array}{c}0.990 \\
\text { (drs) }\end{array}$ \\
\hline & AA & $\begin{array}{l}1.000 \\
\text { (crs) }\end{array}$ & $\begin{array}{l}1.000 \\
\text { (crs) }\end{array}$ & $\begin{array}{c}0.952 \\
\text { (irs) }\end{array}$ & $\begin{array}{l}1.000 \\
\text { (crs) }\end{array}$ & $\begin{array}{c}0.934 \\
\text { (irs) }\end{array}$ & $\begin{array}{c}0.906 \\
\text { (irs) }\end{array}$ & $\begin{array}{c}0.852 \\
\text { (irs) }\end{array}$ & $\begin{array}{c}0.795 \\
\text { (irs) }\end{array}$ & $\begin{array}{c}0.913 \\
\text { (irs) }\end{array}$ & $\begin{array}{c}0.833 \\
\text { (irs) }\end{array}$ \\
\hline \multirow{2}{*}{ Jiangsu } & BA & $\begin{array}{c}0.470 \\
\text { (drs) }\end{array}$ & $\begin{array}{c}0.746 \\
\text { (drs) }\end{array}$ & $\begin{array}{c}0.472 \\
\text { (drs) }\end{array}$ & $\begin{array}{c}0.696 \\
\text { (drs) }\end{array}$ & $\begin{array}{c}0.795 \\
\text { (drs) }\end{array}$ & $\begin{array}{c}0.685 \\
\text { (drs) }\end{array}$ & $\begin{array}{c}0.974 \\
\text { (drs) }\end{array}$ & $\begin{array}{l}1.000 \\
(\mathrm{crs})\end{array}$ & $\begin{array}{c}0.657 \\
\text { (drs) }\end{array}$ & $\begin{array}{c}0.806 \\
\text { (drs) }\end{array}$ \\
\hline & AA & $\begin{array}{c}0.625 \\
\text { (irs) }\end{array}$ & $\begin{array}{c}0.794 \\
\text { (irs) }\end{array}$ & $\begin{array}{c}0.962 \\
\text { (irs) }\end{array}$ & $\begin{array}{l}1.000 \\
\text { (crs) }\end{array}$ & $\begin{array}{l}1.000 \\
\text { (crs) }\end{array}$ & $\begin{array}{l}1.000 \\
\text { (crs) }\end{array}$ & $\begin{array}{l}1.000 \\
\text { (crs) }\end{array}$ & $\begin{array}{l}1.000 \\
\text { (crs) }\end{array}$ & $\begin{array}{l}1.000 \\
\text { (crs) }\end{array}$ & $\begin{array}{c}1.000 \\
\text { (crs) }\end{array}$ \\
\hline \multirow{2}{*}{ Zhejiang } & BA & $\begin{array}{c}0.462 \\
\text { (drs) }\end{array}$ & $\begin{array}{c}0.373 \\
\text { (drs) }\end{array}$ & $\begin{array}{c}0.319 \\
\text { (drs) }\end{array}$ & $\begin{array}{c}0.753 \\
\text { (drs) }\end{array}$ & $\begin{array}{c}0.729 \\
\text { (drs) }\end{array}$ & $\begin{array}{c}0.738 \\
\text { (drs) }\end{array}$ & $\begin{array}{c}0.681 \\
\text { (drs) }\end{array}$ & $\begin{array}{c}0.705 \\
\text { (drs) }\end{array}$ & $\begin{array}{c}0.708 \\
\text { (drs) }\end{array}$ & $\begin{array}{c}0.661 \\
\text { (drs) }\end{array}$ \\
\hline & AA & $\begin{array}{c}0.363 \\
\text { (irs) }\end{array}$ & $\begin{array}{c}0.470 \\
\text { (irs) }\end{array}$ & $\begin{array}{c}0.566 \\
\text { (irs) }\end{array}$ & $\begin{array}{c}0.618 \\
\text { (irs) }\end{array}$ & $\begin{array}{c}0.708 \\
\text { (irs) }\end{array}$ & $\begin{array}{c}0.708 \\
\text { (irs) }\end{array}$ & $\begin{array}{c}0.839 \\
\text { (irs) }\end{array}$ & $\begin{array}{c}0.990 \\
\text { (irs) }\end{array}$ & $\begin{array}{l}1.000 \\
\text { (crs) }\end{array}$ & $\begin{array}{c}0.997 \\
\text { (irs) }\end{array}$ \\
\hline \multirow{2}{*}{ Anhui } & BA & $\begin{array}{c}0.820 \\
\text { (drs) }\end{array}$ & $\begin{array}{c}0.988 \\
\text { (drs) }\end{array}$ & $\begin{array}{c}0.765 \\
\text { (drs) }\end{array}$ & $\begin{array}{c}0.531 \\
\text { (drs) }\end{array}$ & $\begin{array}{c}0.758 \\
\text { (drs) }\end{array}$ & $\begin{array}{l}1.000 \\
\text { (crs) }\end{array}$ & $\begin{array}{l}1.000 \\
\text { (crs) }\end{array}$ & $\begin{array}{l}1.000 \\
\text { (crs) }\end{array}$ & $\begin{array}{l}1.000 \\
\text { (crs) }\end{array}$ & $\begin{array}{c}1.000 \\
\text { (crs) }\end{array}$ \\
\hline & AA & $\begin{array}{c}0.050 \\
\text { (irs) }\end{array}$ & $\begin{array}{c}0.057 \\
\text { (irs) }\end{array}$ & $\begin{array}{c}0.063 \\
\text { (irs) }\end{array}$ & $\begin{array}{c}0.071 \\
\text { (irs) }\end{array}$ & $\begin{array}{c}0.226 \\
\text { (irs) }\end{array}$ & $\begin{array}{c}0.333 \\
\text { (irs) }\end{array}$ & $\begin{array}{c}0.641 \\
\text { (irs) }\end{array}$ & $\begin{array}{c}0.834 \\
\text { (irs) }\end{array}$ & $\begin{array}{l}1.000 \\
\text { (crs) }\end{array}$ & $\begin{array}{c}1.000 \\
\text { (crs) }\end{array}$ \\
\hline \multirow{2}{*}{ Fujian } & BA & $\begin{array}{c}0.978 \\
\text { (drs) }\end{array}$ & $\begin{array}{c}0.996 \\
\text { (drs) }\end{array}$ & $\begin{array}{c}0.846 \\
\text { (drs) }\end{array}$ & $\begin{array}{c}0.928 \\
\text { (drs) }\end{array}$ & $\begin{array}{c}0.908 \\
\text { (drs) }\end{array}$ & $\begin{array}{c}0.869 \\
\text { (drs) }\end{array}$ & $\begin{array}{l}1.000 \\
\text { (crs) }\end{array}$ & $\begin{array}{l}1.000 \\
\text { (crs) }\end{array}$ & $\begin{array}{c}0.978 \\
\text { (drs) }\end{array}$ & $\begin{array}{c}0.990 \\
\text { (drs) }\end{array}$ \\
\hline & AA & $\begin{array}{c}0.725 \\
\text { (irs) }\end{array}$ & $\begin{array}{c}0.720 \\
\text { (irs) }\end{array}$ & $\begin{array}{c}0.729 \\
\text { (irs) }\end{array}$ & $\begin{array}{c}0.683 \\
\text { (irs) }\end{array}$ & $\begin{array}{c}0.696 \\
\text { (irs) }\end{array}$ & $\begin{array}{c}0.834 \\
\text { (irs) }\end{array}$ & $\begin{array}{c}0.634 \\
\text { (irs) }\end{array}$ & $\begin{array}{c}0.748 \\
\text { (irs) }\end{array}$ & $\begin{array}{c}0.846 \\
\text { (irs) }\end{array}$ & $\begin{array}{c}0.851 \\
\text { (irs) }\end{array}$ \\
\hline \multirow{2}{*}{ Jiangxi } & BA & $\begin{array}{c}0.621 \\
\text { (drs) }\end{array}$ & $\begin{array}{c}0.510 \\
\text { (drs) }\end{array}$ & $\begin{array}{c}0.806 \\
\text { (drs) }\end{array}$ & $\begin{array}{c}0.923 \\
\text { (drs) }\end{array}$ & $\begin{array}{c}0.984 \\
\text { (drs) }\end{array}$ & $\begin{array}{c}0.974 \\
\text { (drs) }\end{array}$ & $\begin{array}{c}0.994 \\
\text { (irs) }\end{array}$ & $\begin{array}{c}0.990 \\
\text { (irs) }\end{array}$ & $\begin{array}{c}0.997 \\
\text { (drs) }\end{array}$ & $\begin{array}{c}0.993 \\
\text { (irs) }\end{array}$ \\
\hline & AA & $\begin{array}{c}0.090 \\
\text { (irs) }\end{array}$ & $\begin{array}{c}0.069 \\
\text { (irs) }\end{array}$ & $\begin{array}{c}0.080 \\
\text { (irs) }\end{array}$ & $\begin{array}{c}0.080 \\
\text { (irs) }\end{array}$ & $\begin{array}{c}0.148 \\
\text { (irs) }\end{array}$ & $\begin{array}{c}0.187 \\
\text { (irs) }\end{array}$ & $\begin{array}{c}0.186 \\
\text { (irs) }\end{array}$ & $\begin{array}{c}0.285 \\
\text { (irs) }\end{array}$ & $\begin{array}{c}0.467 \\
\text { (irs) }\end{array}$ & $\begin{array}{c}0.586 \\
\text { (irs) }\end{array}$ \\
\hline \multirow{2}{*}{ Shandong } & BA & $\begin{array}{c}0.522 \\
\text { (drs) }\end{array}$ & $\begin{array}{c}0.422 \\
\text { (drs) }\end{array}$ & $\begin{array}{c}0.520 \\
\text { (drs) }\end{array}$ & $\begin{array}{c}0.765 \\
\text { (drs) }\end{array}$ & $\begin{array}{c}0.837 \\
\text { (drs) }\end{array}$ & $\begin{array}{c}0.810 \\
\text { (drs) }\end{array}$ & $\begin{array}{c}0.905 \\
\text { (drs) }\end{array}$ & $\begin{array}{c}0.865 \\
\text { (drs) }\end{array}$ & $\begin{array}{c}0.807 \\
\text { (drs) }\end{array}$ & $\begin{array}{c}0.884 \\
\text { (drs) }\end{array}$ \\
\hline & AA & $\begin{array}{c}0.505 \\
\text { (irs) }\end{array}$ & $\begin{array}{c}0.535 \\
\text { (irs) }\end{array}$ & $\begin{array}{c}0.666 \\
\text { (irs) }\end{array}$ & $\begin{array}{c}0.746 \\
\text { (irs) }\end{array}$ & $\begin{array}{c}0.881 \\
\text { (irs) }\end{array}$ & $\begin{array}{c}0.856 \\
\text { (irs) }\end{array}$ & $\begin{array}{c}0.908 \\
\text { (irs) }\end{array}$ & $\begin{array}{c}0.921 \\
\text { (irs) }\end{array}$ & $\begin{array}{c}0.904 \\
\text { (irs) }\end{array}$ & $\begin{array}{c}0.963 \\
\text { (irs) }\end{array}$ \\
\hline \multirow{2}{*}{ Henan } & BA & $\begin{array}{c}0.947 \\
\text { (drs) }\end{array}$ & $\begin{array}{c}0.186 \\
\text { (drs) }\end{array}$ & $\begin{array}{c}0.383 \\
\text { (drs) }\end{array}$ & $\begin{array}{c}0.871 \\
\text { (drs) }\end{array}$ & $\begin{array}{c}0.643 \\
\text { (drs) }\end{array}$ & $\begin{array}{c}0.839 \\
\text { (drs) }\end{array}$ & $\begin{array}{c}0.905 \\
\text { (drs) }\end{array}$ & $\begin{array}{c}0.905 \\
\text { (drs) }\end{array}$ & $\begin{array}{l}1.000 \\
\text { (crs) }\end{array}$ & $\begin{array}{c}1.000 \\
\text { (crs) }\end{array}$ \\
\hline & AA & $\begin{array}{c}0.080 \\
\text { (irs) }\end{array}$ & $\begin{array}{c}0.090 \\
\text { (irs) }\end{array}$ & $\begin{array}{c}0.140 \\
\text { (irs) }\end{array}$ & $\begin{array}{c}0.200 \\
\text { (irs) }\end{array}$ & $\begin{array}{c}0.301 \\
\text { (irs) }\end{array}$ & $\begin{array}{c}0.326 \\
\text { (irs) }\end{array}$ & $\begin{array}{c}0.459 \\
\text { (irs) }\end{array}$ & $\begin{array}{c}0.532 \\
\text { (irs) }\end{array}$ & $\begin{array}{l}1.000 \\
\text { (crs) }\end{array}$ & $\begin{array}{c}1.000 \\
\text { (crs) }\end{array}$ \\
\hline \multirow{2}{*}{ Hubei } & BA & $\begin{array}{c}0.505 \\
\text { (irs) }\end{array}$ & $\begin{array}{c}0.259 \\
\text { (drs) }\end{array}$ & $\begin{array}{c}0.481 \\
\text { (drs) }\end{array}$ & $\begin{array}{c}0.824 \\
\text { (drs) }\end{array}$ & $\begin{array}{c}0.871 \\
\text { (drs) }\end{array}$ & $\begin{array}{c}0.935 \\
\text { (drs) }\end{array}$ & $\begin{array}{c}1.000 \\
(\mathrm{crs})\end{array}$ & $\begin{array}{c}0.946 \\
\text { (drs) }\end{array}$ & $\begin{array}{c}0.916 \\
\text { (drs) }\end{array}$ & $\begin{array}{c}0.999 \\
\text { (irs) }\end{array}$ \\
\hline & AA & $\begin{array}{c}0.162 \\
\text { (irs) }\end{array}$ & $\begin{array}{c}0.109 \\
\text { (irs) }\end{array}$ & $\begin{array}{c}0.168 \\
\text { (irs) }\end{array}$ & $\begin{array}{c}0.175 \\
\text { (irs) }\end{array}$ & $\begin{array}{c}0.384 \\
\text { (irs) }\end{array}$ & $\begin{array}{c}0.370 \\
\text { (irs) }\end{array}$ & $\begin{array}{c}0.436 \\
\text { (irs) }\end{array}$ & $\begin{array}{c}0.581 \\
\text { (irs) }\end{array}$ & $\begin{array}{c}0.684 \\
\text { (crs) }\end{array}$ & $\begin{array}{c}0.620 \\
\text { (irs) }\end{array}$ \\
\hline \multirow{2}{*}{ Hunan } & BA & $\begin{array}{c}0.642 \\
\text { (drs) }\end{array}$ & $\begin{array}{c}0.839 \\
\text { (drs) }\end{array}$ & $\begin{array}{c}0.702 \\
\text { (drs) }\end{array}$ & $\begin{array}{c}0.866 \\
\text { (drs) }\end{array}$ & $\begin{array}{c}0.936 \\
\text { (drs) }\end{array}$ & $\begin{array}{c}0.911 \\
\text { (drs) }\end{array}$ & $\begin{array}{l}1.000 \\
\text { (crs) }\end{array}$ & $\begin{array}{l}1.000 \\
\text { (crs) }\end{array}$ & $\begin{array}{l}1.000 \\
\text { (crs) }\end{array}$ & $\begin{array}{c}0.999 \\
\text { (irs) }\end{array}$ \\
\hline & AA & $\begin{array}{c}0.039 \\
\text { (irs) }\end{array}$ & $\begin{array}{c}0.042 \\
\text { (irs) }\end{array}$ & $\begin{array}{c}0.049 \\
\text { (irs) }\end{array}$ & $\begin{array}{c}0.105 \\
\text { (irs) }\end{array}$ & $\begin{array}{c}0.270 \\
\text { (irs) }\end{array}$ & $\begin{array}{c}0.260 \\
\text { (irs) }\end{array}$ & $\begin{array}{c}0.555 \\
\text { (irs) }\end{array}$ & $\begin{array}{c}0.592 \\
\text { (irs) }\end{array}$ & $\begin{array}{c}0.793 \\
\text { (irs) }\end{array}$ & $\begin{array}{c}0.886 \\
\text { (irs) }\end{array}$ \\
\hline \multirow{2}{*}{ Guangdong } & BA & $\begin{array}{c}0.354 \\
\text { (drs) }\end{array}$ & $\begin{array}{l}0.327 \\
\text { (drs) }\end{array}$ & $\begin{array}{c}0.322 \\
\text { (drs) }\end{array}$ & $\begin{array}{l}1.000 \\
\text { (crs) }\end{array}$ & $\begin{array}{c}0.833 \\
\text { (drs) }\end{array}$ & $\begin{array}{l}1.000 \\
\text { (crs) }\end{array}$ & $\begin{array}{l}0.911 \\
\text { (drs) }\end{array}$ & $\begin{array}{l}0.951 \\
\text { (drs) }\end{array}$ & $\begin{array}{l}1.000 \\
\text { (crs) }\end{array}$ & $\begin{array}{c}1.000 \\
\text { (crs) }\end{array}$ \\
\hline & AA & $\begin{array}{l}1.000 \\
(\mathrm{crs})\end{array}$ & $\begin{array}{l}1.000 \\
\text { (crs) }\end{array}$ & $\begin{array}{l}1.000 \\
\text { (crs) }\end{array}$ & $\begin{array}{l}1.000 \\
(\mathrm{crs})\end{array}$ & $\begin{array}{l}1.000 \\
(\mathrm{crs})\end{array}$ & $\begin{array}{l}1.000 \\
(\mathrm{crs})\end{array}$ & $\begin{array}{l}1.000 \\
(\mathrm{crs})\end{array}$ & $\begin{array}{l}1.000 \\
(\mathrm{crs})\end{array}$ & $\begin{array}{l}1.000 \\
\text { (crs) }\end{array}$ & $\begin{array}{c}1.000 \\
\text { (crs) }\end{array}$ \\
\hline \multirow{2}{*}{ Guangxi } & BA & $\begin{array}{c}0.752 \\
\text { (drs) }\end{array}$ & $\begin{array}{c}0.642 \\
\text { (drs) }\end{array}$ & $\begin{array}{c}0.679 \\
\text { (drs) }\end{array}$ & $\begin{array}{c}0.976 \\
\text { (drs) }\end{array}$ & $\begin{array}{c}0.980 \\
\text { (drs) }\end{array}$ & $\begin{array}{c}0.976 \\
\text { (drs) }\end{array}$ & $\begin{array}{c}0.970 \\
\text { (irs) }\end{array}$ & $\begin{array}{c}0.964 \\
\text { (irs) }\end{array}$ & $\begin{array}{c}0.975 \\
\text { (irs) }\end{array}$ & $\begin{array}{c}0.942 \\
\text { (irs) }\end{array}$ \\
\hline & AA & $\begin{array}{c}0.043 \\
\text { (irs) }\end{array}$ & $\begin{array}{c}0.016 \\
\text { (irs) }\end{array}$ & $\begin{array}{c}0.027 \\
\text { (irs) }\end{array}$ & $\begin{array}{c}0.035 \\
\text { (irs) }\end{array}$ & $\begin{array}{c}0.052 \\
\text { (irs) }\end{array}$ & $\begin{array}{c}0.038 \\
\text { (irs) }\end{array}$ & $\begin{array}{c}0.085 \\
\text { (irs) }\end{array}$ & $\begin{array}{c}0.115 \\
\text { (irs) }\end{array}$ & $\begin{array}{c}0.170 \\
\text { (irs) }\end{array}$ & $\begin{array}{c}0.160 \\
\text { (irs) }\end{array}$ \\
\hline \multirow{2}{*}{ Hainan } & BA & $\begin{array}{c}1.000 \\
(\mathrm{crs})\end{array}$ & $\begin{array}{c}1.000 \\
(\mathrm{crs})\end{array}$ & $\begin{array}{c}1.000 \\
(\mathrm{crs})\end{array}$ & $\begin{array}{l}1.000 \\
(\mathrm{crs})\end{array}$ & $\begin{array}{c}1.000 \\
(\mathrm{crs})\end{array}$ & $\begin{array}{c}1.000 \\
(\mathrm{crs})\end{array}$ & $\begin{array}{c}1.000 \\
(\mathrm{crs})\end{array}$ & $\begin{array}{l}1.000 \\
(\mathrm{crs})\end{array}$ & $\begin{array}{l}1.000 \\
(\mathrm{crs})\end{array}$ & $\begin{array}{c}0.966 \\
\text { (irs) }\end{array}$ \\
\hline & AA & $\begin{array}{c}0.002 \\
\text { (irs) }\end{array}$ & $\begin{array}{c}0.001 \\
\text { (irs) }\end{array}$ & $\begin{array}{c}0.005 \\
\text { (irs) }\end{array}$ & $\begin{array}{c}0.003 \\
\text { (irs) }\end{array}$ & $\begin{array}{c}0.045 \\
\text { (irs) }\end{array}$ & $\begin{array}{c}0.022 \\
\text { (irs) }\end{array}$ & $\begin{array}{c}0.070 \\
\text { (irs) }\end{array}$ & $\begin{array}{c}0.116 \\
\text { (irs) }\end{array}$ & $\begin{array}{c}0.141 \\
\text { (irs) }\end{array}$ & $\begin{array}{c}0.150 \\
\text { (irs) }\end{array}$ \\
\hline \multirow{2}{*}{ Chongqing } & BA & $\begin{array}{c}0.934 \\
\text { (drs) }\end{array}$ & $\begin{array}{c}0.516 \\
\text { (drs) }\end{array}$ & $\begin{array}{c}0.631 \\
\text { (drs) }\end{array}$ & $\begin{array}{c}0.725 \\
\text { (drs) }\end{array}$ & $\begin{array}{c}0.973 \\
\text { (drs) }\end{array}$ & $\begin{array}{c}0.925 \\
\text { (drs) }\end{array}$ & $\begin{array}{l}1.000 \\
\text { (crs) }\end{array}$ & $\begin{array}{c}0.993 \\
\text { (irs) }\end{array}$ & $\begin{array}{c}0.999 \\
\text { (irs) }\end{array}$ & $\begin{array}{c}0.992 \\
\text { (irs) }\end{array}$ \\
\hline & AA & $\begin{array}{c}0.076 \\
\text { (irs) }\end{array}$ & $\begin{array}{c}0.083 \\
\text { (irs) }\end{array}$ & $\begin{array}{c}0.088 \\
\text { (irs) }\end{array}$ & $\begin{array}{c}0.134 \\
\text { (irs) }\end{array}$ & $\begin{array}{c}0.235 \\
\text { (irs) }\end{array}$ & $\begin{array}{c}0.227 \\
\text { (irs) }\end{array}$ & $\begin{array}{c}0.413 \\
\text { (irs) }\end{array}$ & $\begin{array}{c}0.349 \\
\text { (irs) }\end{array}$ & $\begin{array}{c}0.519 \\
\text { (irs) }\end{array}$ & $\begin{array}{c}0.530 \\
\text { (irs) }\end{array}$ \\
\hline \multirow{2}{*}{ Sichuan } & BA & $\begin{array}{c}0.815 \\
\text { (drs) }\end{array}$ & $\begin{array}{c}0.675 \\
\text { (drs) }\end{array}$ & $\begin{array}{c}0.458 \\
\text { (drs) }\end{array}$ & $\begin{array}{c}0.664 \\
\text { (drs) }\end{array}$ & $\begin{array}{c}0.973 \\
\text { (drs) }\end{array}$ & $\begin{array}{c}0.672 \\
\text { (drs) }\end{array}$ & $\begin{array}{c}1.000 \\
\text { (crs) }\end{array}$ & $\begin{array}{c}0.999 \\
\text { (drs) }\end{array}$ & $\begin{array}{c}0.947 \\
\text { (drs) }\end{array}$ & $\begin{array}{c}0.984 \\
\text { (drs) }\end{array}$ \\
\hline & AA & $\begin{array}{c}0.302 \\
\text { (irs) }\end{array}$ & $\begin{array}{c}0.344 \\
\text { (irs) }\end{array}$ & $\begin{array}{c}0.466 \\
\text { (irs) }\end{array}$ & $\begin{array}{c}0.419 \\
\text { (irs) }\end{array}$ & $\begin{array}{c}0.584 \\
\text { (irs) }\end{array}$ & $\begin{array}{c}0.447 \\
\text { (irs) }\end{array}$ & $\begin{array}{c}0.552 \\
\text { (irs) }\end{array}$ & $\begin{array}{c}0.816 \\
\text { (irs) }\end{array}$ & $\begin{array}{c}0.873 \\
\text { (irs) }\end{array}$ & $\begin{array}{c}0.899 \\
\text { (irs) }\end{array}$ \\
\hline \multirow{2}{*}{ Guizhou } & BA & $\begin{array}{c}0.865 \\
\text { (drs) }\end{array}$ & $\begin{array}{c}0.224 \\
\text { (drs) }\end{array}$ & $\begin{array}{c}0.330 \\
\text { (drs) }\end{array}$ & $\begin{array}{c}0.691 \\
\text { (drs) }\end{array}$ & $\begin{array}{c}0.835 \\
\text { (drs) }\end{array}$ & $\begin{array}{c}0.924 \\
\text { (drs) }\end{array}$ & $\begin{array}{c}0.926 \\
\text { (drs) }\end{array}$ & $\begin{array}{c}0.997 \\
\text { (drs) }\end{array}$ & $\begin{array}{c}0.999 \\
\text { (drs) }\end{array}$ & $\begin{array}{c}0.990 \\
\text { (irs) }\end{array}$ \\
\hline & AA & $\begin{array}{c}0.050 \\
\text { (irs) }\end{array}$ & $\begin{array}{c}0.060 \\
\text { (irs) }\end{array}$ & $\begin{array}{c}0.102 \\
\text { (irs) }\end{array}$ & $\begin{array}{c}0.114 \\
\text { (irs) }\end{array}$ & $\begin{array}{c}0.143 \\
\text { (irs) }\end{array}$ & $\begin{array}{c}0.188 \\
\text { (irs) }\end{array}$ & $\begin{array}{c}0.192 \\
\text { (irs) }\end{array}$ & $\begin{array}{c}0.312 \\
\text { (irs) }\end{array}$ & $\begin{array}{c}0.393 \\
\text { (irs) }\end{array}$ & $\begin{array}{c}0.418 \\
\text { (irs) }\end{array}$ \\
\hline \multirow{2}{*}{ Yunnan } & BA & $\begin{array}{l}1.000 \\
\text { (crs) }\end{array}$ & $\begin{array}{c}0.371 \\
\text { (drs) }\end{array}$ & $\begin{array}{c}0.458 \\
\text { (drs) }\end{array}$ & $\begin{array}{l}1.000 \\
(\mathrm{crs})\end{array}$ & $\begin{array}{l}1.000 \\
\text { (crs) }\end{array}$ & $\begin{array}{c}0.952 \\
\text { (drs) }\end{array}$ & $\begin{array}{c}0.980 \\
\text { (irs) }\end{array}$ & $\begin{array}{c}0.994 \\
\text { (irs) }\end{array}$ & $\begin{array}{c}0.987 \\
\text { (irs) }\end{array}$ & $\begin{array}{c}0.978 \\
\text { (irs) }\end{array}$ \\
\hline & AA & $\begin{array}{c}0.082 \\
\text { (irs) }\end{array}$ & $\begin{array}{c}0.018 \\
\text { (irs) }\end{array}$ & $\begin{array}{c}0.080 \\
\text { (irs) }\end{array}$ & $\begin{array}{c}0.034 \\
\text { (irs) }\end{array}$ & $\begin{array}{c}0.072 \\
\text { (irs) }\end{array}$ & $\begin{array}{c}0.045 \\
\text { (irs) }\end{array}$ & $\begin{array}{c}0.087 \\
\text { (irs) }\end{array}$ & $\begin{array}{c}0.137 \\
\text { (irs) }\end{array}$ & $\begin{array}{c}0.132 \\
\text { (irs) }\end{array}$ & $\begin{array}{c}0.144 \\
\text { (irs) }\end{array}$ \\
\hline
\end{tabular}




\begin{tabular}{|c|c|c|c|c|c|c|c|c|c|c|c|}
\hline \multirow{2}{*}{ Shaanxi } & BA & $\begin{array}{c}0.525 \\
\text { (drs) }\end{array}$ & $\begin{array}{c}0.550 \\
(\mathrm{drs})\end{array}$ & $\begin{array}{c}0.493 \\
\text { (drs) }\end{array}$ & $\begin{array}{c}0.801 \\
(\mathrm{drs})\end{array}$ & $\begin{array}{c}0.935 \\
\text { (drs) }\end{array}$ & $\begin{array}{c}0.818 \\
\text { (drs) }\end{array}$ & $\begin{array}{c}0.957 \\
\text { (drs) }\end{array}$ & $\begin{array}{c}0.993 \\
\text { (irs) }\end{array}$ & $\begin{array}{c}0.933 \\
\text { (drs) }\end{array}$ & $\begin{array}{c}0.991 \\
\text { (irs) }\end{array}$ \\
\hline & AA & $\begin{array}{c}0.175 \\
\text { (irs) }\end{array}$ & $\begin{array}{c}0.172 \\
\text { (irs) }\end{array}$ & $\begin{array}{c}0.221 \\
\text { (irs) }\end{array}$ & $\begin{array}{c}0.231 \\
\text { (irs) }\end{array}$ & $\begin{array}{c}0.229 \\
\text { (irs) }\end{array}$ & $\begin{array}{c}0.259 \\
\text { (irs) }\end{array}$ & $\begin{array}{c}0.340 \\
\text { (irs) }\end{array}$ & $\begin{array}{c}0.406 \\
\text { (irs) }\end{array}$ & $\begin{array}{c}0.550 \\
\text { (irs) }\end{array}$ & $\begin{array}{c}0.474 \\
\text { (irs) }\end{array}$ \\
\hline \multirow{2}{*}{ Gansu } & BA & $\begin{array}{c}0.529 \\
(\mathrm{drs})\end{array}$ & $\begin{array}{c}0.996 \\
\text { (irs) }\end{array}$ & $\begin{array}{c}0.924 \\
\text { (irs) }\end{array}$ & $\begin{array}{c}0.966 \\
\text { (irs) }\end{array}$ & $\begin{array}{c}0.910 \\
\text { (irs) }\end{array}$ & $\begin{array}{c}0.987 \\
\text { (drs) }\end{array}$ & $\begin{array}{c}0.994 \\
\text { (irs) }\end{array}$ & $\begin{array}{c}0.928 \\
\text { (irs) }\end{array}$ & $\begin{array}{c}0.914 \\
\text { (irs) }\end{array}$ & $\begin{array}{c}0.909 \\
\text { (irs) }\end{array}$ \\
\hline & AA & $\begin{array}{c}0.024 \\
\text { (irs) }\end{array}$ & $\begin{array}{c}0.010 \\
\text { (irs) }\end{array}$ & $\begin{array}{c}0.008 \\
\text { (irs) }\end{array}$ & $\begin{array}{c}0.016 \\
\text { (irs) }\end{array}$ & $\begin{array}{c}0.026 \\
\text { (irs) }\end{array}$ & $\begin{array}{c}0.046 \\
\text { (irs) }\end{array}$ & $\begin{array}{c}0.045 \\
\text { (irs) }\end{array}$ & $\begin{array}{c}0.084 \\
\text { (irs) }\end{array}$ & $\begin{array}{c}0.083 \\
\text { (irs) }\end{array}$ & $\begin{array}{c}0.109 \\
\text { (irs) }\end{array}$ \\
\hline \multirow{2}{*}{ Qinghai } & BA & $\begin{array}{c}1.000 \\
(\mathrm{crs})\end{array}$ & $\begin{array}{c}1.000 \\
(\mathrm{crs})\end{array}$ & $\begin{array}{c}1.000 \\
(\mathrm{crs})\end{array}$ & $\begin{array}{c}0.387 \\
\text { (irs) }\end{array}$ & $\begin{array}{c}0.590 \\
\text { (irs) }\end{array}$ & $\begin{array}{c}1.000 \\
(\mathrm{crs})\end{array}$ & $\begin{array}{l}1.000 \\
(\mathrm{crs})\end{array}$ & $\begin{array}{c}0.162 \\
\text { (irs) }\end{array}$ & $\begin{array}{c}0.989 \\
\text { (irs) }\end{array}$ & $\begin{array}{c}0.490 \\
\text { (irs) }\end{array}$ \\
\hline & AA & $\begin{array}{c}0.001 \\
\text { (irs) }\end{array}$ & $\begin{array}{c}0.011 \\
\text { (irs) }\end{array}$ & $\begin{array}{c}0.011 \\
\text { (irs) }\end{array}$ & $\begin{array}{c}0.000 \\
\text { (irs) }\end{array}$ & $\begin{array}{c}0.006 \\
\text { (irs) }\end{array}$ & $\begin{array}{c}0.005 \\
\text { (irs) }\end{array}$ & $\begin{array}{c}0.005 \\
\text { (irs) }\end{array}$ & $\begin{array}{c}0.001 \\
\text { (irs) }\end{array}$ & $\begin{array}{c}0.006 \\
\text { (irs) }\end{array}$ & $\begin{array}{c}0.003 \\
\text { (irs) }\end{array}$ \\
\hline \multirow{2}{*}{ Ningxia } & BA & $\begin{array}{c}0.946 \\
\text { (irs) }\end{array}$ & $\begin{array}{c}0.999 \\
(\mathrm{crs})\end{array}$ & $\begin{array}{c}0.946 \\
\text { (irs) }\end{array}$ & $\begin{array}{c}0.886 \\
\text { (irs) }\end{array}$ & $\begin{array}{c}0.641 \\
\text { (irs) }\end{array}$ & $\begin{array}{c}0.870 \\
\text { (irs) }\end{array}$ & $\begin{array}{c}0.946 \\
\text { (drs) }\end{array}$ & $\begin{array}{c}0.839 \\
\text { (irs) }\end{array}$ & $\begin{array}{c}1.000 \\
\text { (crs) }\end{array}$ & $\begin{array}{c}0.730 \\
\text { (irs) }\end{array}$ \\
\hline & AA & $\begin{array}{c}0.010 \\
\text { (irs) }\end{array}$ & $\begin{array}{c}0.010 \\
\text { (irs) }\end{array}$ & $\begin{array}{c}0.011 \\
\text { (irs) }\end{array}$ & $\begin{array}{c}0.019 \\
\text { (irs) }\end{array}$ & $\begin{array}{c}0.021 \\
\text { (irs) }\end{array}$ & $\begin{array}{c}0.030 \\
\text { (irs) }\end{array}$ & $\begin{array}{c}0.036 \\
\text { (irs) }\end{array}$ & $\begin{array}{c}0.042 \\
\text { (irs) }\end{array}$ & $\begin{array}{c}0.042 \\
\text { (irs) }\end{array}$ & $\begin{array}{c}0.029 \\
\text { (irs) }\end{array}$ \\
\hline \multirow{2}{*}{ Average value } & $\mathrm{BA}$ & 0.753 & 0.620 & 0.666 & 0.840 & 0.873 & 0.895 & 0.961 & 0.929 & 0.947 & 0.926 \\
\hline & $\mathrm{AA}$ & 0.254 & 0.255 & 0.302 & 0.315 & 0.362 & 0.369 & 0.411 & 0.473 & 0.553 & 0.556 \\
\hline
\end{tabular}

Note: "irs" indicates increasing scale efficiency; "crs" indicates that the scale efficiency is unchanged; "drs" indicates a decline in scale efficiency. "BA" means Before Adjustment, "AA" means After Adjustment

\subsection{Regression analysis of SFA in the second stage}

In order to separate the efficiency values influenced by external environment and random error factors, this paper observes the influence of circumstantial factors and random error respectively by constructing similar SFA models in the second stage. SFA regression analysis is carried out by Frontier software. The relaxation values of each input variable obtained in the first stage are taken as dependent variables, and the selected 6 environmental variables are taken as independent variables to establish regression models to estimate the influence of circumstantial factors on the relaxation values of each input variable. If the regression coefficient is negative, it indicates that the positive change of environmental variables is conducive to reducing the input redundancy of high-tech industries, thus avoiding the loss and waste of input and improving the efficiency of research and development innovation. On the contrary, it will reduce the efficiency level of $\mathrm{R} \& \mathrm{D}$ innovation. The results of SFA regression analysis are shown in Table 6.

Table 6. Estimated results of SFA

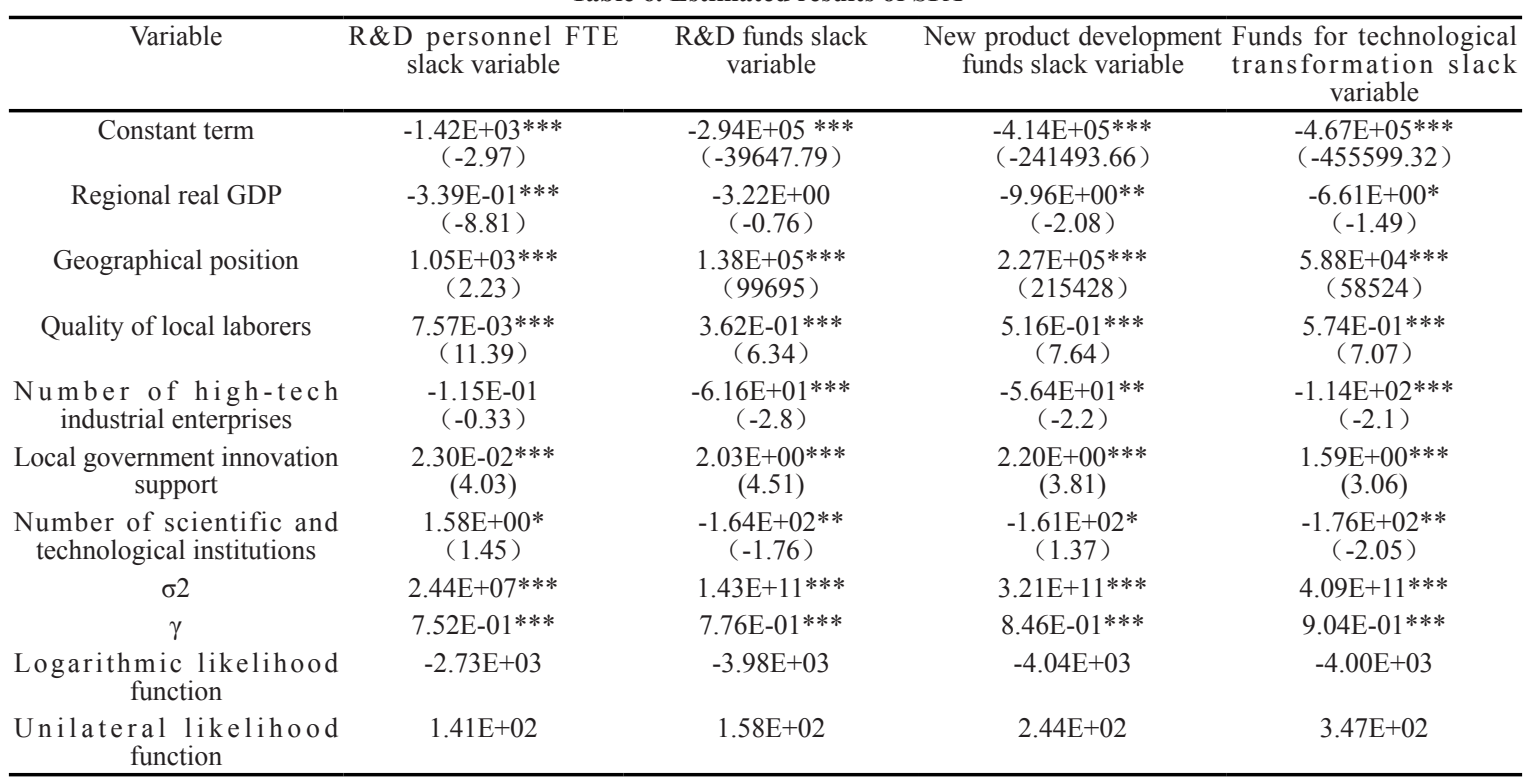

Note: *** means $\mathbf{p}<0.01, * *$ means $\mathbf{p}<0.05, *$ means $\mathbf{p}<0.1$

As can be seen from Table 6, the estimation results $\sigma^{2}$ and $\gamma$ are both significant at the level of $1 \%$, indicating that it is necessary to eliminate the influence of environmental variables in the model. Specifically, it can be seen from the following aspects: (1) Regional actual GDP has a significant negative impact on R\&D personnel's FTE slack, new product development spending slack, and technological transformation spending slack. This shows that the higher the level of regional economic development, the more conducive it is to reduce the redundancy of input variables, and the more 
effective it is to promote the improvement of R\&D innovation efficiency in high-tech industries. (2) Geographical location has a positive effect on all input slack variables at a highly significant level, and compared with other environmental variables, geographical location has a stronger effect on all kinds of innovation input slack. The results show that the eastern region has higher investment redundancy compared with the central and western regions. This is mainly due to the greater demand for innovation in the eastern region, which often spends huge sums of money on research and development innovation, thus easily causing excessive investment in innovation. (3) The influence of regional workers' quality on four types of input slack variables also shows significant positive effects. The higher the number of college students in the region, the higher the quality of workers in the region. However, on the other hand, with the continuous improvement of China's education level, the number of talents engaged in high-tech industries is increasing and even reaching saturation, which to a certain extent increases the redundancy of input variables and reduces the innovation efficiency level of China's high-tech industries. (4) The number of enterprises in high-tech industries has a significant negative impact on the relaxation of R\&D funds, new product development funds and technological transformation funds, which shows that the increase of competition in the industry can effectively reduce the redundancy of capital investment and is beneficial to the improvement of R\&D innovation efficiency. (5) Local government innovation support has significant positive effects on four types of input slack variables, which indicates that the stronger the government's support for high-tech industries, the easier it is to increase the redundancy of each input, and the more unfavorable it is to improve innovation efficiency. This may be because some inappropriate subsidy policies adopted by the government distort the market, undermine the selfregulating function of the market and hinder the improvement of innovation efficiency. (6) The number of scientific and technological institutions has a significant positive effect on R\&D personnel FTE slack variables, while it has a significant negative effect on R\&D funds, new product development funds and technological transformation funds. This shows that although the increase in the number of scientific and technological institutions will increase the redundancy of human capital investment and hinder the improvement of innovation efficiency, it can effectively reduce the waste of R\&D funds, new product development funds and technological transformation funds, thus promoting the improvement of innovation efficiency.

To sum up, environmental variables have significant effects on input redundancy and their effects are different. Therefore, it is necessary to eliminate the effects of circumstantial factors and random factors when studying the true level of innovation efficiency.

\subsection{DEA empirical analysis and malmquist index}

Using DEAP software again, DEA analysis is conducted based on the original output data and the adjusted input variable data. Based on the results shown in Tables 3, 4 and 5 (after adjustment), the estimated outcomes are shown in Table 6.

Overall, the efficiency values before and after the adjustment have changed. The adjusted average technical efficiency is $0.25-0.53$, the average pure technical efficiency is $0.98-0.96$, and the average scale efficiency is $0.254-0.556$.Compared with before adjustment, the average value of technical efficiency in different years has decreased, but the average value of pure technical efficiency has increased significantly, while the average value of pure technical efficiency is relatively stable, approaching 1.The average value of scale efficiency has not only dropped significantly, but also there are only two states after the adjustment: increasing scale returns and constant scale returns. This shows that compared with the situation before the adjustment, China's high-tech industry does not actually have the problems of excessive scale and overcapacity. From a local point of view, the regions where the average value of technical efficiency has increased include Beijing, Shanghai, Jiangsu, Zhejiang, Shandong, Guangdong and Shaanxi, indicating that the low efficiency of these provinces before adjustment is due to circumstantial factors. The average value of comprehensive technical efficiency in other regions has decreased, which indicates that the high level of innovation efficiency shown in these regions is mainly influenced by favorable environment and random factors.

In order to further investigate the changes in total factor productivity of China's high-tech industries, this paper constructs Malmquist index model based on the DEA model adjusted in the third stage. The results are shown in Tables 7 , 8 and 9. The columns in the table are the index changes for every two consecutive years from 2005 to 2014 and the index changes for the whole period from 2005 to 2014. The last two rows are the arithmetic average and geometric average of the index respectively. Generally, geometric mean is used to calculate average ratio and average speed, so this paper uses this value to represent the average growth rate of total factor productivity, technical efficiency index and technical progress index. Table 10 summarizes Malmquist index and its decomposition index of technological innovation in order to meet the needs of total factor productivity of R\&D innovation in various regions in subsequent research. 
Table 7. Changes in total factor productivity index of technological innovation in high-tech industries

\begin{tabular}{|c|c|c|c|c|c|c|c|c|c|c|}
\hline $\begin{array}{r}\text { Year } \\
\text { Provinces }\end{array}$ & $2005 / 2006$ & $2006 / 2007$ & $2007 / 2008$ & $2008 / 2009$ & $2009 / 2010$ & $2010 / 2011$ & $2011 / 2012$ & $2012 / 2013$ & $2013 / 2014$ & $2005 / 2014$ \\
\hline Beijing & 1.144 & 2.691 & 0.999 & 0.958 & 0.952 & 1.049 & 1.181 & 0.871 & 1.087 & 1.141 \\
\hline Tianjin & 1.066 & 0.878 & 0.869 & 0.93 & 0.977 & 0.843 & 1.276 & 1.314 & 0.952 & 0.999 \\
\hline Heibei & 1.299 & 1.169 & 1.444 & 1.48 & 0.8 & 1.473 & 1.207 & 1.287 & 1.262 & 1.251 \\
\hline Shanxi & 2.186 & 2.204 & 0.754 & 1.192 & 1.69 & 1.204 & 1.576 & 1.17 & 0.693 & 1.309 \\
\hline Inner Mongolia & 0.556 & 0.8 & 0.565 & 19.297 & 0.534 & 1.56 & 0.771 & 1.589 & 0.977 & 1.191 \\
\hline Liaoning & 0.817 & 1.451 & 1.498 & 0.93 & 0.801 & 1.588 & 1.015 & 1.049 & 0.933 & 1.085 \\
\hline Jilin & 0.71 & 1.486 & 1.078 & 2.471 & 0.397 & 2.665 & 1.45 & 1.264 & 0.843 & 1.185 \\
\hline Heilongjiang & 0.338 & 1.388 & 1.302 & 1.536 & 0.745 & 2.068 & 1.14 & 1.077 & 1.334 & 1.100 \\
\hline Shanghai & 1.109 & 0.941 & 1.057 & 0.782 & 0.955 & 1.185 & 1.095 & 0.94 & 1.211 & 1.022 \\
\hline Jiangsu & 1.181 & 1.357 & 1.211 & 0.853 & 0.858 & 1.54 & 0.97 & 0.917 & 1.077 & 1.086 \\
\hline Zhejiang & 1.275 & 1.115 & 1.048 & 1.61 & 0.689 & 1.541 & 1.178 & 0.979 & 0.851 & 1.107 \\
\hline Anhui & 1.191 & 1.113 & 1.193 & 3.639 & 1.006 & 2.247 & 1.214 & 1.054 & 1.147 & 1.388 \\
\hline Fujian & 1.009 & 0.999 & 1.01 & 0.894 & 1.088 & 1.078 & 1.02 & 0.939 & 0.937 & 0.995 \\
\hline Jiangxi & 0.953 & 1.111 & 1.04 & 1.829 & 1.136 & 1.214 & 1.326 & 1.498 & 1.309 & 1.246 \\
\hline Shandong & 1.17 & 1.219 & 1.079 & 1.127 & 0.992 & 1.083 & 0.99 & 0.906 & 0.959 & 1.054 \\
\hline Henan & 1.413 & 1.367 & 1.304 & 1.95 & 0.76 & 1.597 & 1.08 & 2.413 & 1.073 & 1.367 \\
\hline Hubei & 0.965 & 1.405 & 1.07 & 2.174 & 0.883 & 1.476 & 1.21 & 1.02 & 0.853 & 1.177 \\
\hline Hunan & 1.212 & 1.071 & 2.098 & 2.905 & 0.785 & 2.483 & 0.983 & 1.219 & 1.074 & 1.394 \\
\hline Guangdong & 1.203 & 1.031 & 0.942 & 1.135 & 1.038 & 1.167 & 0.954 & 1.01 & 1.008 & 1.051 \\
\hline Guangxi & 0.592 & 1.408 & 1.191 & 1.963 & 0.601 & 2.802 & 1.253 & 1.314 & 0.895 & 1.191 \\
\hline Hainan & 1.002 & 3.76 & 0.529 & 18.576 & 0.354 & 3.899 & 1.534 & 1.082 & 1.051 & 1.647 \\
\hline Chongqing & 1.179 & 1.023 & 1.63 & 1.674 & 0.902 & 2.46 & 0.772 & 1.327 & 1.041 & 1.256 \\
\hline Sichuan & 1.18 & 1.337 & 0.94 & 1.273 & 0.604 & 1.635 & 1.65 & 0.904 & 1.112 & 1.134 \\
\hline Guizhou & 1.704 & 1.517 & 0.933 & 1.707 & 0.957 & 1.185 & 1.474 & 1.102 & 1.197 & 1.278 \\
\hline Yunnan & 0.343 & 4.176 & 0.381 & 2.515 & 0.55 & 2.368 & 1.471 & 0.865 & 1.056 & 1.102 \\
\hline Shaanxi & 1.017 & 1.284 & 0.996 & 1.105 & 0.929 & 1.678 & 1.107 & 1.239 & 0.792 & 1.104 \\
\hline Gansu & 0.699 & 0.734 & 1.9 & 1.925 & 1.369 & 1.216 & 1.744 & 0.872 & 1.306 & 1.225 \\
\hline Qinghai & 34.988 & 0.943 & 0.03 & 20.816 & 0.527 & 1.452 & 0.187 & 5.327 & 0.506 & 1.259 \\
\hline Ningxia & 1.232 & 1.087 & 1.807 & 1.065 & 1.281 & 1.608 & 1.077 & 1.642 & 0.376 & 1.150 \\
\hline $\begin{array}{l}\text { Arithmetic } \\
\text { Average }\end{array}$ & 2.232 & 1.451 & 1.100 & 3.459 & 0.868 & 1.702 & 1.169 & 1.317 & 0.997 & 1.189 \\
\hline $\begin{array}{l}\text { Geometry } \\
\text { Average }\end{array}$ & 1.117 & 1.317 & 0.946 & 1.922 & 0.820 & 1.596 & 1.107 & 1.202 & 0.967 & 1.182 \\
\hline
\end{tabular}

Table 8. Changes in technological efficiency index of technological innovation in high-tech industries

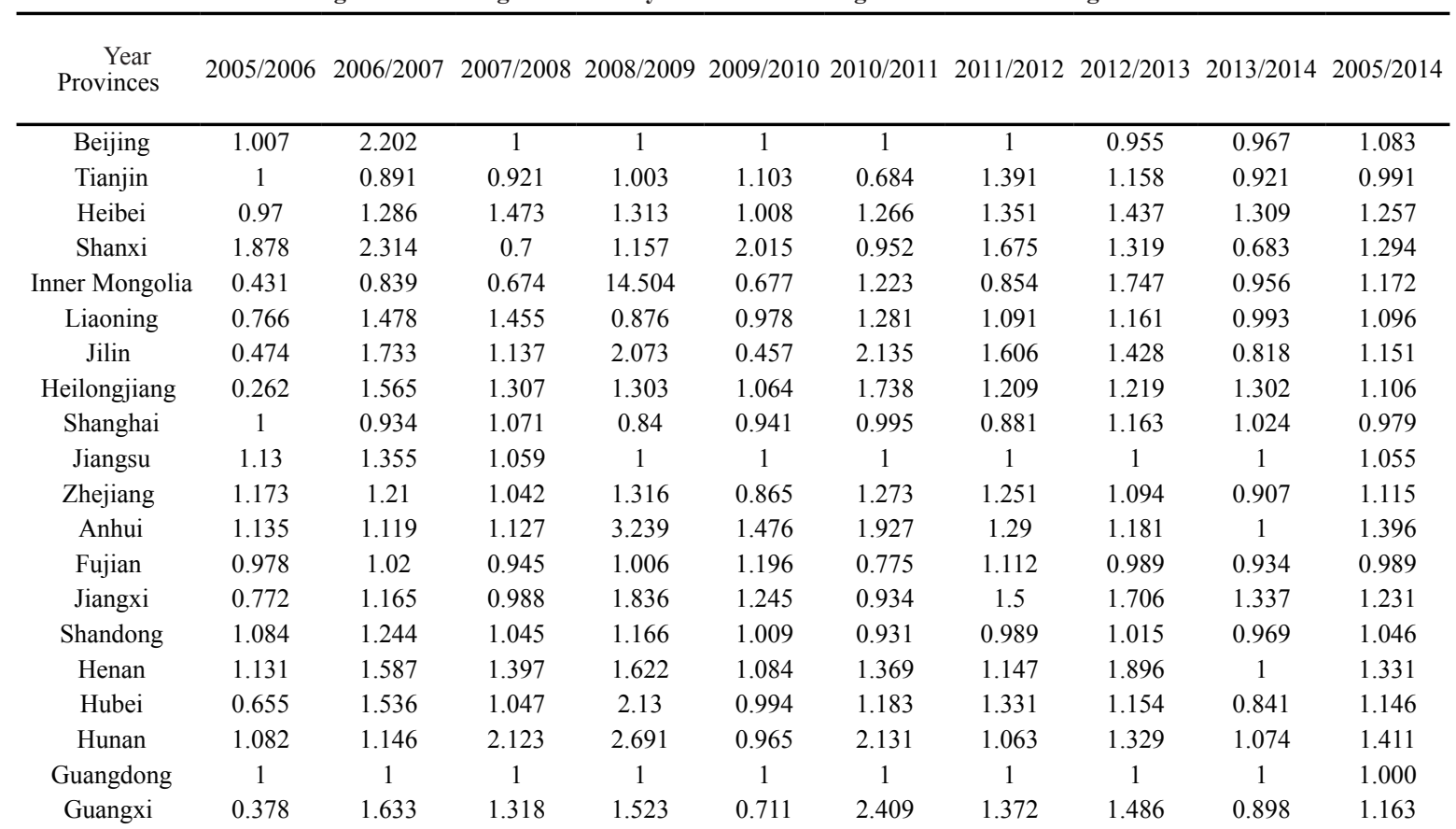




\begin{tabular}{ccccccccccc} 
Hainan & 0.628 & 4.054 & 0.631 & 13.962 & 0.505 & 3.343 & 1.63 & 1.223 & 1.05 & 1.626 \\
Chongqing & 1.097 & 1.065 & 1.508 & 1.814 & 0.976 & 1.805 & 0.857 & 1.495 & 1.019 & 1.248 \\
Sichuan & 1.128 & 1.361 & 0.834 & 1.439 & 0.764 & 1.328 & 1.49 & 1.043 & 1.068 & 1.134 \\
Guizhou & 1.201 & 1.736 & 1.091 & 1.283 & 1.375 & 1.004 & 1.566 & 1.234 & 1.055 & 1.264 \\
Yunnan & 0.215 & 4.558 & 0.417 & 2.147 & 0.649 & 1.998 & 1.572 & 0.975 & 1.057 & 1.070 \\
Shaanxi & 0.891 & 1.335 & 0.99 & 1.011 & 1.106 & 1.328 & 1.052 & 1.407 & 0.827 & 1.088 \\
Gansu & 0.453 & 0.813 & 1.897 & 1.614 & 1.807 & 1.033 & 1.864 & 0.983 & 1.306 & 1.197 \\
Qinghai & 21.919 & 1.016 & 0.036 & 15.646 & 0.732 & 1.198 & 0.199 & 6.003 & 0.496 & 1.232 \\
Ningxia & 1.039 & 1.094 & 1.65 & 1.162 & 1.423 & 1.226 & 1.148 & 1.853 & 0.375 & 1.131 \\
Arithmetic & 1.616 & 1.527 & 1.099 & 2.851 & 1.039 & 1.395 & 1.224 & 1.436 & 0.972 & 1.172 \\
Average & 0.903 & 1.385 & 0.959 & 1.776 & 0.986 & 1.302 & 1.157 & 1.316 & 0.944 & 1.164 \\
\hline
\end{tabular}

Table 9. Changes in technological progress index of technological innovation in high-tech industries

\begin{tabular}{|c|c|c|c|c|c|c|c|c|c|c|}
\hline $\begin{array}{c}\text { Year } \\
\text { Provinces }\end{array}$ & $2005 / 2006$ & $2006 / 2007$ & $2007 / 2008$ & $2008 / 2009$ & $2009 / 2010$ & $2010 / 2011$ & $2011 / 2012$ & $2012 / 2013$ & $2013 / 2014$ & $2005 / 2014$ \\
\hline Beijing & 1.136 & 1.222 & 0.999 & 0.958 & 0.952 & 1.049 & 1.181 & 0.912 & 1.124 & 1.054 \\
\hline Tianjin & 1.066 & 0.986 & 0.943 & 0.928 & 0.886 & 1.231 & 0.918 & 1.135 & 1.033 & 1.009 \\
\hline Heibei & 1.339 & 0.909 & 0.98 & 1.127 & 0.793 & 1.164 & 0.893 & 0.896 & 0.964 & 0.995 \\
\hline Shanxi & 1.164 & 0.953 & 1.077 & 1.03 & 0.839 & 1.265 & 0.941 & 0.887 & 1.015 & 1.011 \\
\hline Inner Mongolia & 1.289 & 0.953 & 0.838 & 1.33 & 0.788 & 1.275 & 0.902 & 0.91 & 1.021 & 1.016 \\
\hline Liaoning & 1.067 & 0.981 & 1.029 & 1.061 & 0.819 & 1.24 & 0.93 & 0.903 & 0.94 & 0.990 \\
\hline Jilin & 1.496 & 0.857 & 0.949 & 1.192 & 0.868 & 1.248 & 0.903 & 0.885 & 1.03 & 1.029 \\
\hline Heilongjiang & 1.29 & 0.887 & 0.996 & 1.179 & 0.701 & 1.19 & 0.943 & 0.883 & 1.025 & 0.995 \\
\hline Shanghai & 1.109 & 1.008 & 0.987 & 0.931 & 1.015 & 1.191 & 1.243 & 0.809 & 1.183 & 1.044 \\
\hline Jiangsu & 1.045 & 1.001 & 1.144 & 0.853 & 0.858 & 1.54 & 0.97 & 0.917 & 1.077 & 1.029 \\
\hline Zhejiang & 1.086 & 0.921 & 1.006 & 1.223 & 0.796 & 1.21 & 0.942 & 0.895 & 0.938 & 0.993 \\
\hline Anhui & 1.049 & 0.995 & 1.058 & 1.124 & 0.682 & 1.166 & 0.941 & 0.892 & 1.147 & 0.994 \\
\hline Fujian & 1.032 & 0.98 & 1.069 & 0.889 & 0.91 & 1.391 & 0.917 & 0.95 & 1.003 & 1.007 \\
\hline Jiangxi & 1.235 & 0.953 & 1.052 & 0.996 & 0.912 & 1.299 & 0.884 & 0.878 & 0.979 & 1.012 \\
\hline Shandong & 1.08 & 0.98 & 1.033 & 0.966 & 0.983 & 1.163 & 1.001 & 0.893 & 0.99 & 1.007 \\
\hline Henan & 1.248 & 0.862 & 0.934 & 1.203 & 0.701 & 1.166 & 0.941 & 1.273 & 1.073 & 1.027 \\
\hline Hubei & 1.473 & 0.915 & 1.023 & 1.021 & 0.888 & 1.248 & 0.91 & 0.884 & 1.014 & 1.027 \\
\hline Hunan & 1.121 & 0.934 & 0.988 & 1.08 & 0.813 & 1.165 & 0.925 & 0.917 & 1 & 0.988 \\
\hline Guangdong & 1.203 & 1.031 & 0.942 & 1.135 & 1.038 & 1.167 & 0.954 & 1.01 & 1.008 & 1.051 \\
\hline Guangxi & 1.567 & 0.862 & 0.904 & 1.289 & 0.846 & 1.163 & 0.913 & 0.884 & 0.997 & 1.025 \\
\hline Hainan & 1.596 & 0.928 & 0.838 & 1.33 & 0.702 & 1.166 & 0.941 & 0.885 & 1.001 & 1.013 \\
\hline Chongqing & 1.075 & 0.96 & 1.081 & 0.923 & 0.924 & 1.363 & 0.901 & 0.888 & 1.021 & 1.006 \\
\hline Sichuan & 1.046 & 0.983 & 1.126 & 0.884 & 0.791 & 1.231 & 1.108 & 0.867 & 1.041 & 1.000 \\
\hline Guizhou & 1.42 & 0.874 & 0.855 & 1.33 & 0.696 & 1.18 & 0.941 & 0.893 & 1.135 & 1.011 \\
\hline Yunnan & 1.596 & 0.916 & 0.913 & 1.171 & 0.847 & 1.185 & 0.936 & 0.888 & 0.998 & 1.030 \\
\hline Shaanxi & 1.142 & 0.962 & 1.006 & 1.092 & 0.841 & 1.263 & 1.051 & 0.881 & 0.957 & 1.014 \\
\hline Gansu & 1.542 & 0.903 & 1.002 & 1.192 & 0.757 & 1.176 & 0.935 & 0.887 & 1 & 1.023 \\
\hline Qinghai & 1.596 & 0.928 & 0.838 & 1.33 & 0.72 & 1.212 & 0.941 & 0.887 & 1.019 & 1.023 \\
\hline Ningxia & 1.186 & 0.993 & 1.095 & 0.917 & 0.9 & 1.312 & 0.938 & 0.887 & 1.005 & 1.017 \\
\hline $\begin{array}{c}\text { Arithmetic } \\
\text { Average }\end{array}$ & 1.252 & 0.953 & 0.990 & 1.093 & 0.837 & 1.228 & 0.960 & 0.916 & 1.025 & 1.015 \\
\hline $\begin{array}{c}\text { Geometry } \\
\text { Average }\end{array}$ & 1.237 & 0.951 & 0.986 & 1.082 & 0.831 & 1.225 & 0.957 & 0.913 & 1.024 & 1.015 \\
\hline
\end{tabular}

Table 10. Malmquist index of technological innovation in high-tech industry and its decomposition

\begin{tabular}{cccccc}
\hline Provinces & TFP & TP & TEC & PTE & SE \\
\hline Beijing & 1.141 & 1.054 & 1.083 & 1.003 & 1.08 \\
Tianjin & 0.999 & 1.008 & 0.991 & 1 & 0.991 \\
Heibei & 1.251 & 0.995 & 1.257 & 1 & 1.257 \\
Shanxi & 1.309 & 1.011 & 1.294 & 1 & 1.294 \\
Inner Mongolia & 1.191 & 1.016 & 1.172 & 1.002 & 1.171 \\
Liaoning & 1.085 & 0.99 & 1.096 & 0.994 & 1.102 \\
Jilin & 1.184 & 1.029 & 1.151 & 0.996 & 1.156 \\
Heilongjiang & 1.101 & 0.995 & 1.106 & 0.999 & 1.107 \\
Shanghai & 1.022 & 1.044 & 0.979 & 0.999 & 0.98
\end{tabular}




\begin{tabular}{cccccc} 
Jiangsu & 1.086 & 1.029 & 1.055 & 1.002 & 1.053 \\
Zhejiang & 1.107 & 0.993 & 1.115 & 1 & 1.114 \\
Anhui & 1.388 & 0.994 & 1.395 & 1 & 1.395 \\
Fujian & 0.995 & 1.007 & 0.989 & 0.972 & 1.018 \\
Jiangxi & 1.246 & 1.012 & 1.231 & 1 & 1.231 \\
Shandong & 1.054 & 1.007 & 1.046 & 0.973 & 1.075 \\
Henan & 1.367 & 1.027 & 1.331 & 1.002 & 1.328 \\
Hubei & 1.177 & 1.027 & 1.146 & 0.992 & 1.155 \\
Hunan & 1.394 & 0.988 & 1.41 & 0.997 & 1.415 \\
Guangdong & 1.05 & 1.05 & 1 & 1 & 1 \\
Guangxi & 1.191 & 1.025 & 1.163 & 1 & 1.163 \\
Hainan & 1.647 & 1.013 & 1.625 & 1 & 1.625 \\
Chongqing & 1.256 & 1.006 & 1.248 & 1.004 & 1.244 \\
Sichuan & 1.134 & 1 & 1.134 & 1.006 & 1.128 \\
Guizhou & 1.278 & 1.011 & 1.264 & 0.993 & 1.272 \\
Yunnan & 1.102 & 1.03 & 1.07 & 1 & 1.071 \\
Shaanxi & 1.104 & 1.014 & 1.088 & 0.979 & 1.112 \\
Gansu & 1.225 & 1.023 & 1.197 & 1.005 & 1.191 \\
Qinghai & 1.26 & 1.023 & 1.232 & 1.003 & 1.229 \\
Ningxia & 1.151 & 1.017 & 1.131 & 1.003 & 1.128 \\
Mean & 1.182 & 1.015 & 1.164 & 0.997 & 1.168 \\
\hline
\end{tabular}

Note: TFP, TP, TEC, PTE and SE in the table respectively represent total factor productivity, technological progress, technological efficiency and pure technological efficiency. And TFP $=$ TP $*$ TEC, TEC $=P$ TE $*$ SE

Generally speaking, the average total factor productivity of R\&D innovation in China's high-tech industries increased from 2005 to 2014, with an increase of 18.2\%. Except that the total factor productivity index changes in 2007/2008, 2009/2010 and 2013/2014 are less than 1, the other annual average is greater than 1, which indicates that the innovation efficiency of China's high-tech industry is on the rise. Among them, the innovation efficiency in 2008/2009 increased the most, reaching $92.2 \%$, while the innovation efficiency in 2009/2010 decreased the fastest, by $18 \%$.Further according to the decomposed indexes, the pure technical efficiency decreased by $0.003 \%$, while the scale efficiency increased by $16.8 \%$. The increase in scale efficiency offset the decrease in pure technical efficiency, thus increasing technical efficiency. This shows that the increase in technical innovation efficiency in China's high-tech industries in recent years is due to the improvement in management level and technical efficiency. In addition, from a local point of view, except for Tianjin, Shanghai and Fujian, where the total factor productivity has not increased significantly, the total factor productivity in other places has increased by different ranges, with Hainan having the largest growth range, reaching a growth rate of $64.7 \%$. Except Hebei, Liaoning, Heilongjiang, Zhejiang, Anhui and Hunan, the index of technological progress in other regions showed slow growth, with Beijing having the largest growth rate, with a growth rate of 5.4\%.

\subsection{Chain multiple mediation effect analysis}

In order to clarify the internal mechanism of China's high-tech industry innovation and "mass entrepreneurship" and find the key to accelerate the process of "double innovation", this paper adopts a multi-chain intermediary effect model to analyze the direct effect and various indirect effects between innovation and entrepreneurship from the overall, regional (east, middle and west) and local (provinces and cities) perspectives.

\subsubsection{Overall and regional}

The overall analysis results of the multiple chain intermediary effect model are shown in Figure 2, and the effect results are shown in Table 11.

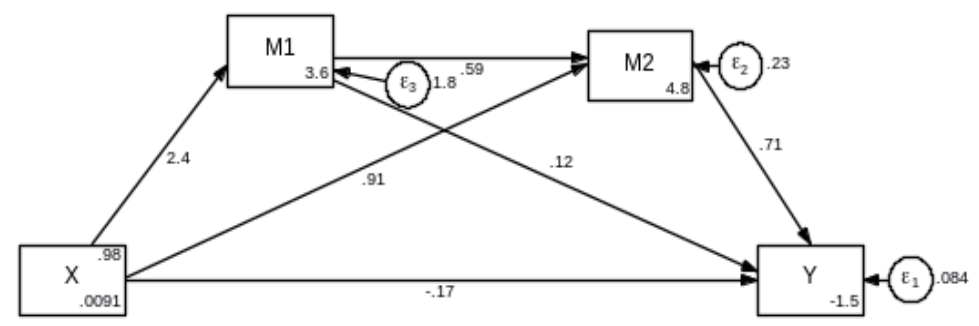

Figure 2. Multiple chain mediating effect based on whole 
Table 11. Results of direct and indirect effects (Overall)

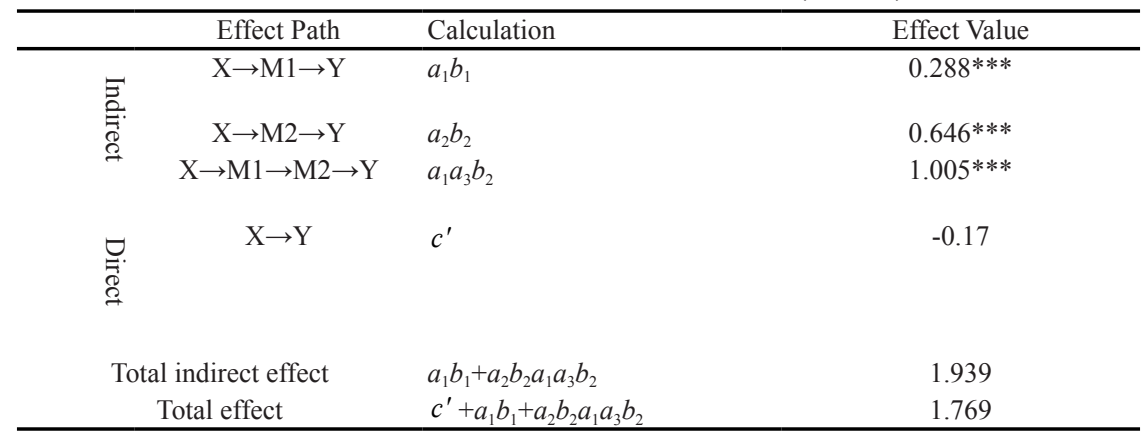

Note: $* * *$ means $\mathbf{p}<0.01, * *$ means $\mathbf{p}<0.05$, * means $\mathbf{p}<0.1$

The analysis results of the east, middle and west regions are shown in the following figure:

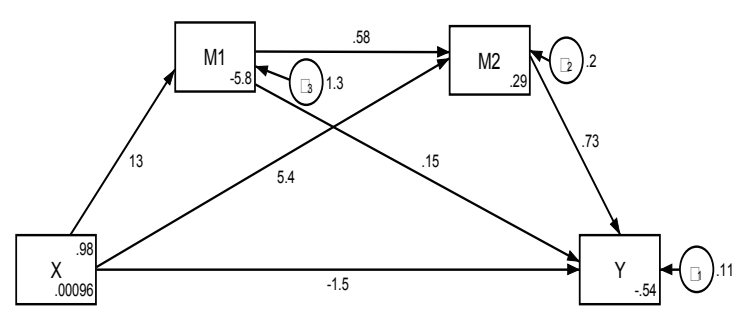

Eastern region effect path

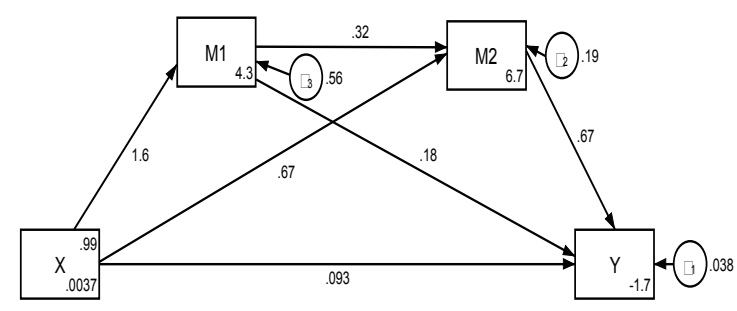

Effect path of central region

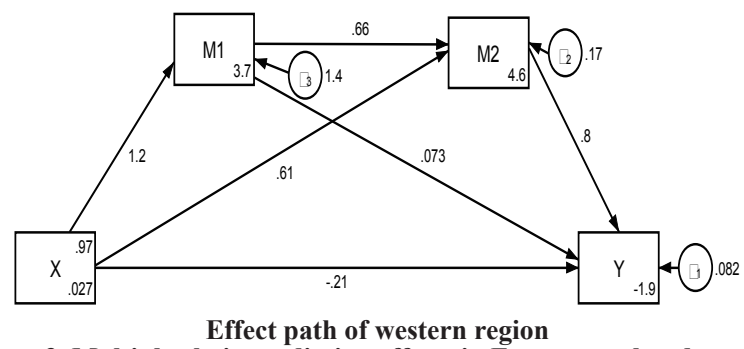

Figure 3. Multiple chain mediating effects in East, central and west regions

Table 12. Direct and indirect effects of East, Middle and West regions and their effect values

\begin{tabular}{|c|c|c|c|c|c|c|}
\hline Region & Direct effect & & Indirect effec & & $\begin{array}{l}\text { Total indirect } \\
\text { utility }\end{array}$ & Total utility \\
\hline East & $-1.5 * *$ & $1.95 * * *$ & $3.942 * *$ & $5.5042 *$ & 11.3962 & 9.8962 \\
\hline In & $0.093 * *$ & $0.288 * *$ & $0.4489 * *$ & $0.3430 * *$ & 1.07994 & 1.17294 \\
\hline West & $-0.21 *$ & $0.792 *$ & $0.488 * *$ & $0.6336 * *$ & 1.9136 & 1.7036 \\
\hline
\end{tabular}

Note: ** means $\mathbf{p}<0.01, * *$ means $\mathbf{p}<0.05, *$ means $\mathbf{p}<0.1$

On the whole, the total effect of innovation on entrepreneurship is 1.769 , that is, the increase of total factor productivity is conducive to the increase of the number of new entrepreneurs. However, from a further perspective, the direct effect of innovation on entrepreneurship is shown as inhibition $\left(c^{\prime}<0\right)$, while the mediating effect through different paths shows a significantly higher promotion effect $\left(a_{1} b_{1}, a_{2} b_{2}, a_{1} a_{3} b_{2}\right.$ are significantly positive).This shows that the promotion effect of innovation on entrepreneurship is not a simple direct effect. The government cannot rely solely on improving innovation efficiency to promote entrepreneurship so as to relieve the pressure on China's labor market. Instead, it should promote entrepreneurship by increasing new ventures and promoting economic growth on the basis of promoting innovation drive.

From the point of view of the east, middle and west regions, the total effect of innovation on entrepreneurship is promotion, and the promotion in the east region is obviously stronger than that in the middle and west regions, which may be caused by the superior geographical position and relatively developed economic level of the east region. The direct 
effects in the east and west are significantly negative, indicating that innovation will play a restraining role in the direct path of entrepreneurship. However, the indirect effects are significantly positive, especially the path in the eastern region, whose influence is much stronger than other paths, which shows that the eastern region mainly promotes entrepreneurship through the path of "innovation $\rightarrow$ enterprise increase $\rightarrow$ economic growth $\rightarrow$ entrepreneurship". For the central region, whether it is a direct or indirect path, the impact of innovation on entrepreneurship always presents a significant positive effect.

\subsubsection{Provinces, cities and regions}

The analysis results of direct effect, indirect effect and total effect of innovation on entrepreneurship in various provinces and cities are shown in Table 13.

Table 13. Direct, indirect and total effects of provinces and cities

\begin{tabular}{|c|c|c|c|c|c|c|c|c|c|c|c|}
\hline & & Direct & & Indirect & & & & Direct & & Indirect & \\
\hline ID & Province & $c^{\prime}$ & $a_{1} b_{1}$ & $a_{2} b_{2}$ & $\mathrm{a}_{1} \mathrm{a}_{3} \mathrm{~b}_{2}$ & ID & Province & $c^{\prime}$ & $a_{1} b_{1}$ & $\mathrm{a}_{2} \mathrm{~b}_{2}$ & $\mathrm{a}_{1} \mathrm{a}_{3} \mathrm{~b}_{2}$ \\
\hline 1 & Beijing & $\begin{array}{c}5.201 * * * \\
(1.757)\end{array}$ & $\begin{array}{l}-6.559 \\
(6.288)\end{array}$ & $\begin{array}{l}-2.633 \\
(4.822)\end{array}$ & $\begin{array}{c}-0.831 * * * \\
(0.173)\end{array}$ & 16 & Henan & $\begin{array}{l}-0.216 \\
(0.557)\end{array}$ & $\begin{array}{l}2.915 \\
(1.946)\end{array}$ & $\begin{array}{l}2.947 \\
(1.832)\end{array}$ & $\begin{array}{c}1.457 * * * \\
(0.114)\end{array}$ \\
\hline 2 & Tianjin & $\begin{array}{l}-4.307 \\
(3.513)\end{array}$ & $\begin{array}{c}3.616 \\
(3.377)\end{array}$ & $\begin{array}{c}11.984 * * \\
(5.399)\end{array}$ & $\begin{array}{l}-0.998 \\
(0.615)\end{array}$ & 17 & Hubei & $\begin{array}{c}0.581 \\
(0.432)\end{array}$ & $\begin{array}{l}-2.694 \\
(2.279)\end{array}$ & $\begin{array}{l}-3.573 \\
(2.799)\end{array}$ & $\begin{array}{c}1.261 * * * \\
(0.472)\end{array}$ \\
\hline 3 & Hebei & $\begin{array}{c}1.264 \\
(2.751)\end{array}$ & $\begin{array}{c}2.260 \\
(4.549)\end{array}$ & $\begin{array}{c}1.628 \\
(3.650)\end{array}$ & $\begin{array}{c}0.794 * * * \\
(0.180)\end{array}$ & 18 & Hunan & $\begin{array}{c}0.309 \\
(0.966)\end{array}$ & $\begin{array}{c}3.751 \\
(4.610)\end{array}$ & $\begin{array}{c}3.298 \\
(3.883)\end{array}$ & $\begin{array}{c}1.950 * * * \\
(0.145)\end{array}$ \\
\hline 4 & Shanxi & $\begin{array}{c}0.862 * * * \\
(0.103)\end{array}$ & $\begin{array}{l}-0.406 \\
(1.834)\end{array}$ & $\begin{array}{c}1.251 \\
(2.184)\end{array}$ & $\begin{array}{c}-2.682 * * * \\
(0.732)\end{array}$ & 19 & Guangdong & $\begin{array}{c}-4.674 * * \\
(1.930)\end{array}$ & $\begin{array}{c}9.218 \\
(6.795)\end{array}$ & $\begin{array}{c}9.520 \\
(7.017)\end{array}$ & $\begin{array}{l}0.982 * * \\
(0.456)\end{array}$ \\
\hline 5 & $\begin{array}{l}\text { I } \mathrm{n} \mathrm{n} \mathrm{e} \mathrm{r} \\
\text { Mongolia }\end{array}$ & $\begin{array}{l}-0.451 \\
(0.180)\end{array}$ & $\begin{array}{c}0.303 \\
(0.596)\end{array}$ & $\begin{array}{c}0.257 \\
(0.883)\end{array}$ & $\begin{array}{c}2.882 * * \\
(1.433)\end{array}$ & 20 & Guangxi & $\begin{array}{l}-0.333 \\
(0.516)\end{array}$ & $\begin{array}{c}1.835 \\
(3.313)\end{array}$ & $\begin{array}{c}3.344 \\
(3.186)\end{array}$ & $\begin{array}{l}1.176^{*} \\
(0.639)\end{array}$ \\
\hline 6 & Liaoning & $\begin{array}{l}-0.680 \\
(0.486)\end{array}$ & $\begin{array}{c}2.317 \\
(3.019)\end{array}$ & $\begin{array}{l}-0.502 \\
(2.617)\end{array}$ & $\begin{array}{l}-0.418 \\
(0.452)\end{array}$ & 21 & Hainan & $\begin{array}{c}-0.785 * * * \\
(0.223)\end{array}$ & $\begin{array}{c}0.596 \\
(0.799)\end{array}$ & $\begin{array}{c}1.846 \\
(1.243)\end{array}$ & $\begin{array}{l}-1.546 \\
(1.133)\end{array}$ \\
\hline 7 & Jilin & $\begin{array}{l}1.114 * \\
(0.607)\end{array}$ & $\begin{array}{l}-4.547 \\
(3.793)\end{array}$ & $\begin{array}{l}-1.757 \\
(5.084)\end{array}$ & $\begin{array}{l}1.941 * * \\
(0.939)\end{array}$ & 22 & Chongqing & $\begin{array}{c}0.326 \\
(0.732)\end{array}$ & $\begin{array}{c}6.575 \\
(4.528)\end{array}$ & $\begin{array}{c}7.843 \\
(5.252)\end{array}$ & $\begin{array}{c}1.216^{* * * *} \\
(0.219)\end{array}$ \\
\hline 8 & Heilongjiang & $\begin{array}{l}-0.506 \\
(0.613)\end{array}$ & $\begin{array}{c}0.007 \\
(0.264)\end{array}$ & $\begin{array}{c}5.044 * * * \\
(1.948)\end{array}$ & $\begin{array}{l}-0.014 \\
(0.504)\end{array}$ & 23 & Sichuan & $\begin{array}{c}0.092 \\
(0.512)\end{array}$ & $\begin{array}{l}-2.002 \\
(2.406)\end{array}$ & $\begin{array}{c}0.041 \\
(1.602)\end{array}$ & $\begin{array}{c}1.050 * * * \\
(0.324)\end{array}$ \\
\hline 9 & Shanghai & $\begin{array}{c}1.899 \\
(1.190)\end{array}$ & $\begin{array}{c}2.565 \\
(4.491)\end{array}$ & $\begin{array}{c}1.379 \\
(4.168)\end{array}$ & $\begin{array}{c}-0.555^{*} * \\
(0.226)\end{array}$ & 24 & Guizhou & $\begin{array}{c}0.299 \\
(0.949)\end{array}$ & $\begin{array}{c}-0.035 \\
(0.357)\end{array}$ & $\begin{array}{c}2.873 \\
(3.699)\end{array}$ & $\begin{array}{c}0.114 \\
(0.767)\end{array}$ \\
\hline 10 & Jiangsu & $\begin{array}{c}0.342 \\
(0.337)\end{array}$ & $\begin{array}{l}-2.641 \\
(5.668)\end{array}$ & $\begin{array}{c}0.969 \\
(5.130)\end{array}$ & $\begin{array}{c}0.885 * * * \\
(0.189)\end{array}$ & 25 & Yunnan & $\begin{array}{c}-1.977^{*} \\
(1.170)\end{array}$ & $\begin{array}{c}0.098 \\
(0.624)\end{array}$ & $\begin{array}{c}-2.534 \\
(4.542)\end{array}$ & $\begin{array}{c}-0.214 \\
(0.907)\end{array}$ \\
\hline 11 & Zhejiang & $\begin{array}{c}-1.569 \\
(1.558)\end{array}$ & $\begin{array}{c}0.370 \\
(2.733)\end{array}$ & $\begin{array}{c}9.835 * * \\
(4.488)\end{array}$ & $\begin{array}{l}-0.067 \\
(0.497)\end{array}$ & 26 & Shaanxi & $\begin{array}{c}2.643 \\
(3.316)\end{array}$ & $\begin{array}{l}-1.189 \\
(5.848)\end{array}$ & $\begin{array}{c}2.710 \\
(5.332)\end{array}$ & $\begin{array}{c}3.571 * * * \\
(0.694)\end{array}$ \\
\hline 12 & Anhui & $\begin{array}{c}0.355 \\
(1.166)\end{array}$ & $\begin{array}{c}3.088 \\
(3.405)\end{array}$ & $\begin{array}{c}5.097 \\
(3.229)\end{array}$ & $\begin{array}{c}0.775 * * * \\
(0.085)\end{array}$ & 27 & Gansu & $\begin{array}{l}-0.189 \\
(0.375)\end{array}$ & $\begin{array}{l}1.510 \\
(2.146)\end{array}$ & $\begin{array}{l}-2.794 \\
(2.800)\end{array}$ & $\begin{array}{c}0.831 * * \\
(0.392)\end{array}$ \\
\hline 13 & Fujian & $\begin{array}{c}-5.648 * * * \\
(1.088)\end{array}$ & $\begin{array}{c}2.330 \\
(4.915)\end{array}$ & $\begin{array}{c}17.260 * * \\
(7.653)\end{array}$ & $\begin{array}{c}2.214 * * * \\
(0.837)\end{array}$ & 28 & Qinghai & $\begin{array}{c}0.056 \\
(0.049)\end{array}$ & $\begin{array}{c}0.206 \\
(0.190)\end{array}$ & $\begin{array}{c}0.112 * \\
(0.067)\end{array}$ & $\begin{array}{c}0.361 * * \\
(0.170)\end{array}$ \\
\hline 14 & Jiangxi & $\begin{array}{c}2.282 \\
(1.500)\end{array}$ & $\begin{array}{l}-2.912 \\
(7.217)\end{array}$ & $\begin{array}{c}0.205 \\
(4.388)\end{array}$ & $\begin{array}{c}1.012 * * * \\
(0.111)\end{array}$ & 29 & Ningxia & $\begin{array}{l}-0.550 \\
(0.710)\end{array}$ & $\begin{array}{c}0.652 \\
(0.917)\end{array}$ & $\begin{array}{c}0.186 \\
(1.056)\end{array}$ & $\begin{array}{c}0.795 \\
(0.513)\end{array}$ \\
\hline 15 & Shandong & $\begin{array}{c}0.184 \\
(1.825)\end{array}$ & $\begin{array}{l}-4.029 \\
(10.165)\end{array}$ & $\begin{array}{c}3.385 \\
(7.460)\end{array}$ & $\begin{array}{c}1.338 * * * \\
(0.352)\end{array}$ & & & & & & \\
\hline
\end{tabular}

Note: * * * means $\mathrm{p}<0.01, * *$ means $\mathrm{p}<0.05, *$ means $\mathrm{p}<0.1$

The direct effects of Beijing, Shanxi and Jilin are significantly positive, while the direct effects of Yunnan, Hainan, Guangdong and Fujian are significantly negative, while the direct effects of most other provinces are not significant. On the contrary, in terms of indirect effects, most provinces have significant positive effects, while only Beijing, Shanxi and Shanghai have negative effects.

\section{Conclusions and policy recommendations}

This paper decomposes total factor productivity on the basis of DEA model excluding environment and random factors and then analyzes the influencing process and mechanism among variables by using chain multiple intermediary effect model and further analyzes the relationship between innovation and entrepreneurship in high-tech industries. The research confirms: (1) Before circumstantial factors and random interference are eliminated, the comprehensive technical efficiency and scale efficiency of R\&D in China's high-tech industry are obviously overestimated, while the pure technical efficiency is underestimated. Therefore, it is necessary to eliminate environmental and random factors and increase the credibility of the research. (2) No matter from the overall or partial analysis, the total effect of innovation on 
entrepreneurship has always been positive, that is, promoting "people-to-people innovation" is conducive to promoting "mass entrepreneurship." (3) In most cases, innovation does not directly promote entrepreneurship but indirectly promotes entrepreneurship through a micro-macro combination path.

\section{Relevant policy recommendations}

Generally speaking, the government can encourage China's high-tech industry to explore new market areas and gain competitive advantages by formulating relevant industrial policies and promoting innovation, thus providing motive force for innovation drive. Through continuous entrepreneurship, innovative achievements will be continuously transformed into real productive forces to promote the sustainable development of high-tech industries and social economy. We should strengthen the combination of "production, study, and research" and focus on improving the "double-creation" mechanism and the "double-creation" environment.

First of all, as the direct effect of innovation on entrepreneurship is positive in Beijing, Shanxi, and Jilin, the government and relevant local departments can appropriately increase the amount of financial allocation for research and development of high-tech products and conquering high-end technologies, strengthen the government's financial support, and give full play to the policy's guidance and support for innovation by strengthening the government's innovation subsidies and tax incentives, thus realizing a "double harvest" of innovation and entrepreneurship on the basis of effectively improving the innovation efficiency of high-tech industries in the region. Secondly, in Fujian, Guangdong, Hainan, and Yunnan, the government should make great efforts to promote the development of other industries while developing high-tech industries, so that the local advantageous industries and characteristic industries can develop continuously and steadily and all kinds of industries will be put together. Thirdly, in Tianjin, Heilongjiang, Zhejiang, Fujian, and Qinghai, the government should actively promote economic development. While making great efforts to improve GDP, it should also pay attention to the per capita level of GDP, scientific and technological content, and green degree and strive to provide a good macroenvironment for promoting "double innovation" by correctly grasping the "three improvements." Finally, in Beijing, Shanghai, and Shanxi, the number of new high-tech enterprises should be controlled to ensure that a reasonable market structure can not only bring into play economies of scale but also promote effective competition, thus effectively stimulating the improvement of innovation efficiency and entrepreneurship scale.

\section{References}

[1] Gui Huang Bao. Spatial Econometric Analysis of Innovation Efficiency and Its Influencing Factors in China's Hightech Industry. Economic Geography. 2014; (06): 100-107.

[2] Han Jing. Research on Innovation Efficiency of China's High-tech Industry-Empirical Analysis Based on SFA Method. Scientific Research. 2010; (03): 467-472.

[3] Xiao renqiao, Qian Li, Chen Zhongwei. Research on innovation efficiency of China's high-tech industry and its influencing factors. Management Science. 2012; (05): 85-98.

[4] Chiu, Y., C. Huang, Y. Chen. The R\&D value-chain efficiency measurement for high-tech industries in China. Asia Pacific Journal of Management. 2012; 29(4): 989-1006.

[5] Dai kui-zao, Liu you-Jin. The impact of marketization on innovation efficiency and industry differences-based on the empirical test of China's high-tech industry. Financial Research. 2013; 39 (5): 4-16.

[6] Wu Yanbing. R\&D Stock, Knowledge Function and Production Efficiency. Economics (Quarterly). 2006; (3): 11291156.

[7] Chen Yanying, Yang Wenlu. knowledge workers' entrepreneurial entry and $r \& d$ incentives for incumbent enterprisesevidence from China's high-tech industry. Scientific Research. 2012; (11): 1707-1714.

[8] Cao Xiaohong, Cai Li, Miao Shujuan. Study on Decision Mechanism of Imitation Entrepreneurship Based on Hightech Industrial Clusters. Scientific Research. 2008; (04): 739-748.

[9] Wang Chongming, Xue Yuanhao. Theoretical Construction and Empirical Analysis of Intellectual Property Entrepreneurship: A Multi-case Study Based on High-tech Enterprises. Journal of Zhejiang University (Humanities and Social Sciences Edition). 2014; (03): 58-70.

[10] Franco, A.M., Filson, D. Spin-outs: knowledge diffusion through employee mobility. The RAND Journal of Economics. 2006; 37(4): 841-860.

[11] Aldrich,H., Zimmer, C. Entrepreneurship through social networks. The art and Science of Entrepreneurship. 1986; 22: $3-23$.

[12] Lester, R.H., Certo, S.T., Dalton, C.M. Initial public offering investor valuations: an examination of top management 
team prestige and environmental uncertainty. Journal of Small Business Management. 2006; 44(1): 1-26.

[13] Chen Bin, Xu Weixiang, Wang Qingxi. Summary of Research on Innovation, Entrepreneurship and Innovation Cluster Development. Scientific and Technological Progress and Countermeasures. 2014; (09): 157-160.

[14] Jin Bei, Xie xiaoxia. Entrepreneurship and innovation mechanism of high-tech industry in the United States and its enlightenment. Management World. 2001(04): 63-70.

[15] Fried H O. Accounting for environmental effects and statistical noise in data envelopment analysis. Journal of Productivity Analysis. 2002; 17:157-174.

[16] Preacher, K. J., Hayes, A. F. Asymptotic and Resampling Strategies for Assessing and Comparing Indirect Effects in Multiple Mediator Models. Behavior Research Methods. 2008; 40: 879-891.

[17] Griliches Z. Patent Statistics as Economic Indicators: A Survey. Journal of Economic Literature. 1990; 28: 16611707. 